\title{
Gain of Regularity for the KP-II Equation
}

\author{
JULIE L. LEVANDOSKY
}

ABSTRACT. In this paper we study the smoothness properties of solutions to the KP-II equation,

$$
\begin{aligned}
& u_{t x}+u_{x x x x}+u_{x x}+u_{y y}+\left(u u_{x}\right)_{x}=0 \\
& u(x, y, 0)=\varphi(x, y) .
\end{aligned}
$$

We show that the equation's dispersive nature leads to a gain in regularity for the solution. In particular, if the initial data $\varphi$ possesses certain minimal regularity and sufficient decay at infinity, then the solution $u(t) \in C^{\infty}\left(\mathbb{R}^{2}\right)$ for $t>0$. More specifically, if $\varphi$ satisfies

$$
\int_{\mathbb{R}^{2}}\left\{\varphi^{2}+\varphi_{x x x}^{2}+\left(\partial_{x}^{-1} \varphi_{y y}\right)^{2}+x_{+}^{L} \varphi^{2}+x_{+}^{L} \varphi_{x}^{2}\right\} d x d y<+\infty
$$

for all integers $L \geq 0$, then there exists a unique solution $u(t) \in$ $C^{\infty}\left(\mathbb{R}^{2}\right)$ for $0<t \leq T$ where $T$ is the existence time of the solution. Thus the singularities in the initial data instantly disappear.

1. IntRoduction. In the late 1800 s the $\mathrm{KdV}$ equation was derived as a model for water wave propagation in shallow water with weak dispersive and weak nonlinear effects. In 1970, Kadomtsev \& Petviashvili [KP] derived a two-dimensional analog

$$
u_{t x}+u_{x x x x}+u_{x x}+\varepsilon u_{y y}+\left(u u_{x}\right)_{x}=0,
$$

where $\varepsilon=\mp 1$, now called the KP-I and KP-II equations respectively. Whereas the $\mathrm{KdV}$ equation models strictly one-dimensional wave propagation, the 
KP equation (1.1) models wave propagation with weak two-dimensional effects. In addition to being used as a model for the evolution of surface waves $[\mathrm{AC}]$, the $\mathrm{KP}$ equation has also been proposed as a model for internal waves in straits or channels of varying depth and width [Sn], [DLW]. The KP equation has also been studied as a model for ion-acoustic wave propagation in isotropic media [PY].

Recently, certain results concerning the Cauchy problem for the KP-I and KP-II equations have appeared. In particular, for the KP-II equation, Faminskii $[\mathrm{Fa}$ ] proved the global existence of weak solutions. Subsequently, Bourgain [Bo] proved that the KP-II equation is globally well-posed for initial data in $H^{s}\left(\mathbb{R}^{2}\right), s \geq 0$. His method is based on an analysis of multiple Fourier series. Here we use different methods to prove a complementary result, showing that if the initial data possesses sufficient decay at spatial infinity, then it is in fact smoother than its initial data.

Other recent work on the KP equations includes the following. Ukai [Uk] proved local well-posedness for both the KP-I and KP-II equations for initial data in $H^{s}\left(\mathbb{R}^{2}\right), s \geq 3$, while Saut [Sa2] proved some local existence results for generalized KP equations. Saut also proved a mild local smoothing property for the KP-II equation. Here we show an infinite gain in regularity if the initial data decays appropriately at infinity.

Recently a number of results concerning gain of regularity for various nonlinear evolution equations have appeared. This paper uses the ideas of Cohen [Co], Kato [Ka], Craig and Goodman [CG], and Craig, Kappeler, and Strauss [CKS] in their respective studies of generalized KdV equations. Kato, motivated by work of Cohen, showed that solutions to the KdV equation are in $C^{\infty}$ for initial data in $L^{2}$ with the weight function $1+e^{\sigma x}, \sigma>0$. Craig, Kappeler, and Strauss expanded on this method in their treatment of highly generalized $\mathrm{KdV}$ equations. Their method allowed them to treat dispersive $\mathrm{KdV}$-type equations with fairly general nonlinear terms. In addition, they quantified the gain in regularity of the solution in terms of the decay at infinity of the initial data.

In attempting to prove the gain of regularity result for the KP-II equation, we are confronted with several obstacles not present in the KdV case. First, we need to consider the direction of propagation of the singularities. For the KdV equation, with only one spatial dimension it is well-known that the singularities all travel in the same direction, specifically to the left. In that case, a nonsymmetric weight function is chosen, which decays as $x \rightarrow-\infty$. By looking at the symbol associated with the KP-II equation, we see that for $t>0$, the bicharacteristics in our case span the entire open left 
half-plane. We hypothesize that the singularities of the solution will travel along these bicharacteristics and, thus, travel in a range of directions. We are able to show, however, that it suffices to choose a weight function depending only on $x$ and $t$ and not on $y$ to prove the gain in regularity. In particular, in order to show the correlation between the decay at infinity of the initial data and the gain in regularity of the solution, we choose the weight function to behave roughly like a power of $x$ for $x>1$ and as a decaying exponential for $x<-1$. With this choice, we are able to show how the gain of derivatives comes at the expense of powers of $x$ for $x>1$.

A second difficulty arising in the proof for the KP-II equation concerns the Sobolev embedding estimates. In one spatial dimension, i.e.-as in the case for the $\mathrm{KdV}$ equation, we have the embedding $H^{1}(\mathbb{R}) \subset L^{\infty}(\mathbb{R})$. We cannot use the same estimate for the KP-II equation, however, as $H^{1}\left(\mathbb{R}^{2}\right) \nsubseteq$ $L^{\infty}\left(\mathbb{R}^{2}\right)$.

\section{Remarks.}

(i) It is known that both the KP-I and KP-II equations have an infinite number of conserved quantities. In particular,

$$
\partial_{t}\left(\int_{\mathbb{R}^{2}}\left(u_{x}^{2} \pm\left(\partial_{x}^{-1} u_{y}\right)^{2}-\frac{1}{3} u^{3}\right) d x d y\right)=0
$$

for the KP-I and KP-II equations, respectively. It is interesting that although the KP-I equation has the positive sign, it is, in fact, the KP-II equation for which there is the gain in regularity. As we will point out later, our method does not show any improved regularity for solutions to the KP-I equation at all. In spite of appearances, the lack of positivity in (1.2) for KP-II does not disturb our proof. However, it does prevent the use of unweighted weak energy estimates.

(ii) The technique we use is not specific to the given nonlinear term. Consequently, we have been able to prove similar results for a wide class of nonlinear dispersive equations in two spatial dimensions. These results will appear in a subsequent paper.

We now turn to a special case of our main theorem on the gain of regularity for the KP-II equation,

$$
\begin{aligned}
& u_{t x}+u_{x x x x}+u_{x x}+u_{y y}+\left(u u_{x}\right)_{x}=0 \\
& u(x, y, 0)=\varphi(x, y) .
\end{aligned}
$$


Before proceeding, we need to introduce the space of functions in which we are considering solutions. We define

$$
X^{0}\left(\mathbb{R}^{2}\right) \equiv\left\{f \mid f, \xi^{3} \hat{f}, \frac{\eta^{2}}{\xi} \hat{f} \in L^{2}\left(\mathbb{R}^{2}\right)\right\},
$$

equipped with the natural norm. On the space $D \equiv\{f \mid(1 / \xi) \hat{f}(\xi, \eta) \in$ $\left.L^{2}\left(\mathbb{R}^{2}\right)\right\}$, we define the operator $\partial_{x}^{-1}$ by $\widehat{\partial_{x}^{-1} f} \equiv 1 /(i \xi) \hat{f}$. Therefore, in particular, we can write the norm of $X^{0}$ as

$$
\|f\|_{X^{0}} \equiv\left(\int_{\mathbb{R}^{2}} f^{2}+f_{x x x}^{2}+\left(\partial_{x}^{-1} f_{y y}\right)^{2}\right)^{1 / 2}
$$

On this space of functions $X^{0}\left(\mathbb{R}^{2}\right)$, it makes sense to rewrite (1.3) as

$$
\begin{aligned}
& u_{t}+u_{x x x}+u_{x}+\partial_{x}^{-1} u_{y y}+u u_{x}=0 \\
& u(x, y, 0)=\varphi(x, y)
\end{aligned}
$$

and consider weak solutions $u \in X^{0}$.

Gain of Regularity Theorem. Let $u$ be a solution of $(1.6)$ in $\mathbb{R}^{2} \times[0, T]$ such that for all integers $L \geq 0$,

$$
\begin{array}{r}
\sup _{0 \leq t \leq T} \int_{\mathbb{R}^{2}}\left\{u^{2}+u_{x x x}^{2}+\left(\partial_{x}^{-1} u_{y y}\right)^{2}\right\} d x d y<+\infty \\
\sup _{0 \leq t \leq T} \int_{\mathbb{R}^{2}}\left(1+x_{+}\right)^{L}\left(u^{2}+u_{x}^{2}\right) d x d y<+\infty,
\end{array}
$$

then our solution $u(t) \in C^{\infty}\left(\mathbb{R}^{2}\right)$ for $0<t \leq T$.

In Section 2, we state the above theorem in fuller generality as Theorem 2.1. Here we will give an overview of the proof. We begin by approximating our initial data $\varphi$ by smooth data $\varphi^{(n)}$. In Section 3, we show that for each smooth $\varphi^{(n)}$ there exists a smooth solution $u^{(n)}$ satisfying (1.7) for a time $\mathrm{T}$ depending only on

$$
\int_{\mathbb{R}^{2}}\left\{\varphi^{2}+\varphi_{x x x}^{2}+\left(\partial_{x}^{-1} \varphi_{y y}\right)^{2}\right\} d x d y<+\infty .
$$

In Section 4, we use a priori estimates to show that our solution $u^{(n)}$ also satisfies (1.8) for that same time $T$ as long as

$$
\int_{\mathbb{R}^{2}}\left(1+x_{+}\right)^{L}\left(\varphi^{2}+\varphi_{x}^{2}\right) d x d y<+\infty
$$


Once we have a smooth solution, we are able to use our other a priori estimates, proven in Section 2, along with an inductive argument, to show the gain in regularity. We can then use convergence arguments to show that a subsequence of $\left\{u^{(n)}\right\}$ converges to $u$.

The idea for the proof of the gain in regularity is the following. For the first step of the induction, we multiply equation (1.3) by $2 \xi u_{x}$ where $\xi$ is our weight function, to be specified later, and integrate over $\mathbb{R}^{2}$. Upon doing so, we get the following inequality:

$$
\begin{aligned}
& \partial_{t} \int \xi u_{x}^{2}+3 \int \xi_{x} u_{x x}^{2}+\int \xi_{x} u_{y}^{2} \\
& \quad \leq \int \xi_{t} u_{x}^{2}+\int \xi_{x x x} u_{x}^{2}+\int \xi_{x} u_{x}^{2}+\left|2 \int \xi u u_{x} u_{x x}\right|,
\end{aligned}
$$

where $\int=\int_{\mathbb{R}^{2}} d x d y$. Notice the second and third terms on the left-hand side. Assuming $\xi, \xi_{x}>0$, these terms have positive signs, thus, allowing us to prove a "gain" in regularity. We continue this procedure inductively. On each step, $\beta$, of the induction, we take $\alpha$ derivatives of the KP-II equation rewritten in the form (1.6) above, where $\alpha_{1}+2 \alpha_{2}=\beta$. We then multiply the differentiated equation by $2 \xi\left(\partial^{\alpha} u\right)$ where $\partial^{\alpha} \equiv \partial_{x}^{\alpha_{1}} \partial_{y}^{\alpha_{2}}$ and $\xi=\xi_{\beta}$ is our weight function to be described below. Integrating over $\mathbb{R}^{2}$, we arrive at the following inequality,

$$
\begin{aligned}
\partial_{t} \int \xi\left(\partial^{\alpha} u\right)^{2}+3 \int \xi_{x}\left(\partial^{\alpha} u_{x}\right)^{2}+\int \xi_{x}\left(\partial^{\alpha}\left(\partial_{x}^{-1} u_{y}\right)\right)^{2} \\
\leq \int \xi_{t}\left(\partial^{\alpha} u\right)^{2}+\int \xi_{x x x}\left(\partial^{\alpha} u\right)^{2}+\int \xi_{x}\left(\partial^{\alpha} u\right)^{2} \\
+\left|2 \int \xi \partial^{\alpha}\left(u u_{x}\right)\left(\partial^{\alpha} u\right)\right|
\end{aligned}
$$

By the nature of the equation, on each level, we gain twice as many $x$ derivatives as $y$ derivatives. We continue this procedure as long as $\xi_{x}=\left(\xi_{\beta}\right)_{x}$ is positive.

Choice of weight function. As mentioned earlier, we will be using nonsymmetric weight functions. In particular, we will be using weight functions $\xi(x, t) \in C^{\infty}$ which behave roughly like powers of $x$ for $x>1$ and decay exponentially for $x<-1$. For each step of the induction, therefore, we decrease our weight function by one power of $\mathrm{x}$, thus showing the correlation between the regularity of the solution and the decay at infinity of the initial data. In particular, we define our weight classes as follows. We say that a 
positive $C^{\infty}$ function $\xi(x, t)$ belongs to the weight class $W_{\sigma, i, k}$, where $\sigma \geq 0$, $k \geq 0$ if $\xi(x, t)$ satisfies the following:

$$
\begin{aligned}
0<c_{1} \leq t^{-k} e^{-\sigma x} \xi(x, t) & \leq c_{2} \quad \text { for } x<-1, \\
0<c_{1} \leq t^{-k} x^{-i} \xi(x, t) & \leq c_{2} \quad \text { for } x>1, \\
\frac{t\left|\partial_{t} \xi\right|+\left|\partial_{x}^{j} \xi\right|}{\xi} & \leq c_{3} \quad \text { in } \mathbb{R} \times[0, T] \text { for all } j .
\end{aligned}
$$

Thus $\xi$ looks like $t^{k}$ as $t \rightarrow 0$, like $x^{i}$ as $x \rightarrow+\infty$, and like $e^{\sigma x}$ as $x \rightarrow-\infty$.

As mentioned above, we have two sets of a priori estimates. The first, proven in Section 4, is used to start our induction. In this case, we show that for $\varphi$ satisfying (1.9) and (1.10), our unique solution $u$ found in Section 3 satisfies (1.7) and (1.8). In Section 2 we use a priori estimates to prove the "gain" in regularity. The fundamental difference is that in Section 2 our weight functions decay exponentially as $x \rightarrow-\infty$, while in Section 4 our weight functions are approximately constant as $x \rightarrow-\infty$. Consequently, in Section 2, to bound terms of the form,

$$
\left|\int_{0}^{T} \int_{\{x<-1\} \times \mathbb{R}} t^{k} e^{\sigma x}\left(\partial^{r} u\right)\left(\partial^{s} u_{x}\right)\left(\partial^{\alpha} u\right)\right|,
$$

on the $\beta$ th step of the induction, we can share the exponential power among the three factors and use the bounds on

$$
\begin{aligned}
& \sup _{0 \leq t \leq T} \int_{\{x<-1\} \times \mathbb{R}} t^{k} e^{\tilde{\sigma} x}\left(\partial^{v} u\right)^{2} \\
& \quad+\int_{0}^{T} \int_{\{x<-1\} \times \mathbb{R}} t^{k} e^{\tilde{\sigma} x}\left[\left(\partial^{v} u_{x}\right)^{2}+\left(\partial^{v}\left(\partial_{x}^{-1} u_{y}\right)\right)^{2}\right]
\end{aligned}
$$

for $v_{1}+2 v_{1} \leq \beta-1, \tilde{\sigma}>0$ arbitrary, from the previous step of the induction. In Section 4, however, because $\xi$ is approximately constant for $x<-1$, we are looking for bounds on terms of the form

$$
\left|\int_{0}^{T} \int_{\{x<-1\} \times \mathbb{R}} c\left(\partial^{r} u\right)\left(\partial^{s} u_{x}\right)\left(\partial^{\alpha} u\right)\right| .
$$

In this case, we cannot use the a priori knowledge on the last two terms in (1.16). Instead, we can only use the a priori bounds on

$$
\sup _{0 \leq t \leq T} \int \xi\left(\partial^{v} u\right)^{2}
$$


for $v_{1}+2 v_{2} \leq \beta-1$.

In other words, on the $(\beta-1)$ st step of the induction for the proof of Lemma 4.2, we are able to show that

$$
\begin{aligned}
& \sup _{0 \leq t \leq T} \int_{\{x<-1\} \times \mathbb{R}}\left(\partial^{v} u\right)^{2} \\
& \quad+\int_{0}^{T} \int_{\{x<-1\} \times \mathbb{R}} e^{\sigma x}\left[\left(\partial^{v} u_{x}\right)^{2}+\left(\partial^{v}\left(\partial_{x}^{-1} u_{y}\right)\right)^{2}\right]<+\infty,
\end{aligned}
$$

for $v_{1}+2 v_{2} \leq \beta-1, \sigma>0$ arbitrary. Unfortunately, when trying to bound terms of the form (1.17), on the $\beta$ th step of the induction, we cannot use the bounds on the second two terms in (1.18). Consequently, the proof of Lemma 4.2 is slightly more technical than the proof of Lemma 2.2 in the region $\{x<-1\} \times \mathbb{R}$.

Notation. As in (1.4) for the definition of $X^{0}$, we define the space of functions $X^{N}\left(\mathbb{R}^{2}\right)$ for $N \geq 0$ as follows

$$
\begin{aligned}
& X^{N}\left(\mathbb{R}^{2}\right) \\
\equiv & \left\{f \mid f \in L^{2}\left(\mathbb{R}^{2}\right), \mathcal{F}^{-1}\left(\xi^{3} \hat{f}\right) \in H^{N}\left(\mathbb{R}^{2}\right), \mathcal{F}^{-1}\left(\frac{\eta^{2}}{\xi} \hat{f}\right) \in H^{N}\left(\mathbb{R}^{2}\right)\right\},
\end{aligned}
$$

equipped with the norm

$$
\|f\|_{X^{N}\left(\mathbb{R}^{2}\right)}^{2}=\int\left[f^{2}+\sum_{|\alpha| \leq N}\left\{\left(\partial^{\alpha} f_{x x x}\right)^{2}+\left(\partial^{\alpha}\left(\partial_{x}^{-1} f_{y y}\right)\right)^{2}\right\}\right] d x d y
$$

where $\alpha=\left(\alpha_{1}, \alpha_{2}\right) \in \mathbb{Z}^{+} \times \mathbb{Z}^{+}$.

We introduce the following notation for our weighted Sobolev-type spaces. Let $\tilde{H}^{\beta}\left(W_{\sigma, i, k}\right)$ be the space of functions with finite norm

$$
\|f\|_{\tilde{H}^{\beta}\left(W_{\sigma, i, k}\right)}^{2}=\int \sum_{\alpha_{1}+2 \alpha_{2} \leq \beta}\left(\partial^{\alpha} f\right)^{2}|\xi(x, t)| d x d y
$$

for any $\xi(x, t) \in W_{\sigma, i, k}, \beta \geq 0$, and $0 \leq t \leq T$. We note that although the norm above depends on $\xi$, all choices of $\xi$ in this class lead to equivalent norms. With the same notation, let $L^{p}\left([0, T] ; \tilde{H}^{\beta}\left(W_{\sigma, i, k}\right)\right)$ be the space of functions with finite norm

$$
\|f\|_{L^{p}\left([0, T] ; \tilde{H}^{\beta}\left(W_{\sigma, i, k}\right)\right)}^{p}=\int_{0}^{T}\left\{\int \sum_{\alpha_{1}+2 \alpha_{2} \leq \beta}\left(\partial^{\alpha} f\right)^{2}|\xi(x, t)| d x d y\right\}^{p / 2} d t
$$


for $\beta \geq 0$, where $\xi(x, t) \in W_{\sigma, i, k}$. In addition, we define the following spaces,

$$
\bar{W}_{\sigma, i, k}=\cup_{j<i} W_{\sigma, j, k}
$$

and

$$
L^{p}\left(\tilde{H}^{s}\left(\bar{W}_{\sigma, i, k}\right)\right)=\cup_{j<i} L^{p}\left(\tilde{H}^{s}\left(W_{\sigma, j, k}\right)\right) .
$$

We shall use the last spaces only in the case when $i=-1$. For simplicity, let

$$
Z_{L} \equiv X^{0}\left(\mathbb{R}^{2}\right) \cap \tilde{H}^{1}\left(W_{0, L, 0}\right) .
$$

With this notation, $Z_{L}$ consists of those functions $u$ such that

$$
\|u\|_{\mathcal{Z}_{L}}^{2} \equiv \int\left\{\xi\left(u^{2}+u_{x}^{2}\right)+u_{x x x}^{2}+\left(\partial_{x}^{-1} u_{y y}\right)^{2}\right\} d x d y<+\infty
$$

for some $\xi \in W_{0, L, 0}$.

2. GAIN OF REGULARITY. In this section we state our main theorem on the gain of regularity for weak solutions $u \in X^{0}\left(\mathbb{R}^{2}\right)$ of the KP-II equation,

$$
\begin{aligned}
& u_{t}+u_{x x x}+u_{x}+\partial_{x}^{-1} u_{y y}+u u_{x}=0 \\
& u(x, y, 0)=\varphi(x, y)
\end{aligned}
$$

We give an outline of the proof as well as the proof of the main estimates used in the inductive argument for the gain in regularity theorem.

Theorem 2.1. Let $T>0$ and let $u$ be the solution of (2.1) in the region $[0, T] \times \mathbb{R}^{2}$ such that $u \in L^{\infty}\left([0, T] ; Z_{L}\right)$ for some integer $L \geq 2$. Then

$$
\begin{array}{r}
\sup _{0 \leq t \leq T} \int \xi_{\beta}\left(\partial^{\alpha} u\right)^{2} d x d y<+\infty, \\
\int_{0}^{T} \int \eta_{\beta}\left(\partial^{\alpha} u_{x}\right)^{2} d x d y d t<+\infty, \\
\int_{0}^{T} \int \eta_{\beta}\left(\partial^{\alpha}\left(\partial_{x}^{-1} u_{y}\right)\right)^{2} d x d y d t<+\infty,
\end{array}
$$

for $0 \leq \beta \leq L+1$, where

$$
\begin{aligned}
& \left.\beta=\alpha_{1}+2 \alpha_{2}, \quad \alpha_{1}, \alpha_{2}\right) \in \mathbb{Z}^{+} \times \mathbb{Z}^{+},
\end{aligned}
$$

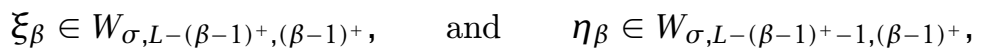

$\sigma>0$ arbitrary, with the exception that for $\beta=L+1, W_{\sigma,-1, L}$ is replaced by $\bar{W}_{\sigma,-1, L}$. 
The proof of Theorem 2.1 relies on a series of lemmas and theorems to follow. We will give the proof in detail at the end of Section 4. Here we provide an outline of the proof.

Outline of proof. We begin by approximating our initial data $\varphi$ by a sequence of functions, $\left\{\varphi^{(n)}\right\}$ in $C_{0}^{\infty}\left(\mathbb{R}^{2}\right)$. We show in Section 3 that there exists a sequence of solutions $\left\{\boldsymbol{u}^{(n)}\right\}$ which exist for a time $T$ depending only on the norm of $\varphi^{(n)}$ in $Z_{L}$. In Section 4 , we prove a persistence property of the initial data showing that for initial data $\varphi$ in a weighted Sobolev space, the solution $u$ found in Section 3 lies in the same weighted Sobolev space. We use this fact to start the inductive argument of our proof.

The inductive argument proceeds as follows. On each level $\beta$ of the induction, we derive a priori estimates for smooth solutions. For each step $\beta$ of the induction, we take $\alpha$ derivatives of (2.1), where $\alpha_{1}+2 \alpha_{2}=\beta$. We multiply our differentiated equation by $2 \xi_{\beta}\left(\partial^{\alpha} u\right)$ and integrate over $\mathbb{R}^{2}$. Upon doing so, we arrive at the following inequality,

$$
\begin{aligned}
& \partial_{t} \int \xi\left(\partial^{\alpha} u\right)^{2}+3 \int \xi_{x}\left(\partial^{\alpha} u_{x}\right)^{2}+\int \xi_{x}\left(\partial^{\alpha}\left(\partial_{x}^{-1} u_{y}\right)\right)^{2} \\
\leq & \int \xi_{t}\left(\partial^{\alpha} u\right)^{2}+\int \xi_{x x x}\left(\partial^{\alpha} u\right)^{2}+\int \xi_{x}\left(\partial^{\alpha} u\right)^{2}+\left|2 \int \xi \partial^{\alpha}\left(u u_{x}\right)\left(\partial^{\alpha} u\right)\right|,
\end{aligned}
$$

where $\xi=\xi_{\beta}$. Using Lemma 2.2, we show that on each level $\beta$ of the induction, the terms on the right-hand side of (2.5) are bounded by our inductive hypothesis, thus proving bounds for the terms on the left-hand side of (2.5). For example, for $\beta=2$, we use the a priori estimates proven in Lemma 2.2, which show that for weight functions $\xi \in W_{\sigma, L-1,2}$ and $\eta \in$ $W_{\sigma, L-2,2}$ and smooth solutions $u^{(n)}$,

$$
\begin{aligned}
& \sup _{0 \leq t \leq T} \int \xi\left(u_{x x}^{(n)}\right)^{2}+\int_{0}^{T} \int \eta\left(u_{x x x}^{(n)}\right)^{2}+\int_{0}^{T} \int \eta\left(u_{x y}^{(n)}\right)^{2} \leq C, \\
& \sup _{0 \leq t \leq T} \int \xi\left(u_{y}^{(n)}\right)^{2}+\int_{0}^{T} \int \eta\left(u_{x y}^{(n)}\right)^{2}+\int_{0}^{T} \int \eta\left(\partial_{x}^{-1} u_{y y}^{(n)}\right)^{2} \leq C,
\end{aligned}
$$

where $C$ depends only on the norm of $u^{(n)} \in L^{\infty}\left([0, T] ; Z_{L}\right)$ and a weighted norm of $u$ for which we proved a bound in Section 4 . We continue this procedure inductively. On each level, we decrease our weight functions $\xi$ and $\eta$ by one power of $x$ and use the result from Lemma 2.2 which shows that

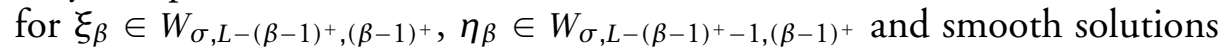
$u^{(n)}$,

$$
\sup _{0 \leq t \leq T} \int \xi_{\beta}\left(\partial^{\alpha} u^{(n)}\right)^{2}+\int_{0}^{T} \int \eta_{\beta}\left(\partial^{\alpha} u_{x}^{(n)}\right)^{2}+\int_{0}^{T} \int \eta_{\beta}\left(\partial^{\alpha}\left(\partial_{x}^{-1} u_{y}^{(n)}\right)\right)^{2} \leq C,
$$


where $C$ depends only the weighted norm of lower-order derivatives of $u$. In particular, these norms have been bounded by the previous step of the induction. As our constant $C$ does not depend on $n$, we are able to pass to the limit and prove our theorem.

We now provide the lemmas and theorems which are used in the proof of Theorem 2.1. At the end of Section 4, we piece these together in the formal proof of Theorem 2.1.

Lemma 2.2. (Main estimates) For $u$, a solution of (2.1), sufficiently smooth and with sufficient decay at infinity,

$$
\sup _{0 \leq t \leq T} \int \xi_{\beta}\left(\partial^{\alpha} u\right)^{2}+\int_{0}^{T} \int \eta_{\beta}\left(\partial^{\alpha} u_{x}\right)^{2}+\int_{0}^{T} \int \eta_{\beta}\left(\partial^{\alpha}\left(\partial_{x}^{-1} u_{y}\right)\right)^{2} \leq C
$$

for $2 \leq \beta \leq L+1$, where $\beta=\alpha_{1}+2 \alpha_{2}, \alpha=\left(\alpha_{1}, \alpha_{2}\right) \in \mathbb{Z}^{+} \times \mathbb{Z}^{+}, \xi_{\beta} \in$ $W_{\sigma, L-(\beta-1)^{+},(\beta-1)^{+}}, \eta_{\beta} \in W_{\sigma, L-(\beta-1)^{+}-1,(\beta-1)^{+}}$, and $C$ depends only on $\|u\|_{X^{0}}$ and the following:

$$
\begin{aligned}
& \sup _{0 \leq t \leq T} \int \xi_{v}\left(\partial^{\gamma} u\right)^{2} d x d y, \\
& \int_{0}^{T} \int \eta_{v}\left(\partial^{\gamma} u_{x}\right)^{2} d x d y d t, \\
& \int_{0}^{T} \int \eta_{v}\left(\partial^{\gamma}\left(\partial_{x}^{-1} u_{y}\right)\right)^{2} d x d y d t,
\end{aligned}
$$

where $\gamma=\left(\gamma_{1}, \gamma_{2}\right) \in \mathbb{Z}^{+} \times \mathbb{Z}^{+}, v=\gamma_{1}+2 \gamma_{2} \leq \beta-1, \xi_{v} \in W_{\sigma, L-(v-1)^{+},(v-1)^{+}}$ and $\eta_{v} \in W_{\sigma, L-(v-1)^{+}-1,(v-1)^{+}}$for $\sigma>0$ arbitrary.

The idea of the proof is the following. We would like to bound terms on the left-hand side of (2.8) in terms of integrals of the same form, but with a lower number of derivatives. In particular, we hope to bound the lefthand side of (2.8) in terms of (2.9)-(2.11). In our inductive argument for the proof of Theorem 2.1, these lower order terms will be bounded on the previous step of the induction. In order to prove these a priori estimates we need to change our weight function $\xi$ on each level $\beta$ by decreasing $\xi$ by one power of $x$ for $x>1$ and increasing by one power of $t$, thus, explaining the choice of $\xi_{\beta}$ and $\eta_{\beta}$ indicated above.

In order to prove some of these estimates, we will use anisotropic Sobolev estimates. These are known and can be found in [BIN], so here we just provide a simple proof of one that we will use frequently. 
Lemma 2.3. For $\alpha, \beta>1,1 / \alpha+1 / \beta<1, u \in L^{2}\left(\mathbb{R}^{2}\right)$,

$$
|u(x, y)|_{L^{\infty}} \leq C\left(\int\left(1+|\xi|^{\alpha}+|\eta|^{\beta}\right)|\hat{u}|^{2} d \xi d \eta\right)^{1 / 2} .
$$

Proof. The proof follows from writing $u$ in terms of its inverse Fourier transform and using the fact that

$$
\left(\int_{\mathbb{R}^{2}} \frac{1}{1+|\xi|^{\alpha}+|\eta|^{\beta}} d \xi d \eta\right)<+\infty
$$

for $\alpha, \beta$ satisfying our hypothesis.

In particular, we have the following corollary.

\section{Corollary 2.4.}

$$
|u(x, y)|_{L^{\infty}} \leq C\left(\int u^{2}+u_{x x}^{2}+u_{y}^{2}\right)^{1 / 2} .
$$

\section{Proof of Lemma 2.2.}

For each $\beta$, we take $\alpha$ derivatives of (2.1), where $\alpha_{1}+2 \alpha_{2}=\beta$, multiply our differentiated equation by $2 \xi_{\beta}\left(\partial^{\alpha} u\right)$ where we take

$$
\xi_{\beta}(x, t)=\int_{-\infty}^{x} \eta_{\beta}(z, t) d z \quad \text { for } \eta_{\beta} \in W_{\sigma, L-(\beta-1)^{+}-1,(\beta-1)^{+}},
$$

and integrate over $\mathbb{R}^{2} \times[0, t]$ for $0 \leq t \leq T$. Upon doing so, we conclude that,

$$
\begin{gathered}
\int \xi(\cdot, t)\left(\partial^{\alpha} u\right)^{2}+\int_{0}^{t} \int \xi_{x}\left(\partial^{\alpha} u_{x}\right)^{2}+\int_{0}^{t} \int \xi_{x}\left(\partial^{\alpha}\left(\partial_{x}^{-1} u_{y}\right)\right)^{2} \\
\leq \int \xi(\cdot, 0)\left(\partial^{\alpha} \varphi\right)^{2}+\int_{0}^{t} \int \xi_{t}\left(\partial^{\alpha} u\right)^{2}+\int_{0}^{t} \int \xi_{x x x}\left(\partial^{\alpha} u\right)^{2} \\
+\int_{0}^{t} \int \xi_{x}\left(\partial^{\alpha} u\right)^{2}+C\left|\int_{0}^{t} \int \xi\left(\partial^{\alpha} u\right) \partial^{\alpha}\left(u u_{x}\right)\right|
\end{gathered}
$$

where $\xi \equiv \xi_{\beta}$. Now using the fact that $\xi_{x}, \xi_{t} \leq C \xi$ and $\xi_{\beta}(\cdot, 0)=0$ for $\beta \geq 2$, we get the following identity,

$$
\begin{gathered}
\int \xi(\cdot, t)\left(\partial^{\alpha} u\right)^{2}+\int_{0}^{t} \int \xi_{x}\left(\partial^{\alpha} u_{x}\right)^{2}+\int_{0}^{t} \int \xi_{x}\left(\partial^{\alpha}\left(\partial_{x}^{-1} u_{y}\right)\right)^{2} \\
\leq C \int_{0}^{t} \int \xi\left(\partial^{\alpha} u\right)^{2}+C\left|\int_{0}^{t} \int \xi\left(\partial^{\alpha} u\right) \partial^{\alpha}\left(u u_{x}\right)\right| .
\end{gathered}
$$


On each level $\beta$, we must look for bounds on the remainder term,

$$
\left|\int_{0}^{t} \int \xi\left(\partial^{\alpha} u\right) \partial^{\alpha}\left(u u_{x}\right)\right|
$$

in terms of (2.9)-(2.11). In particular, we must be careful in breaking up the above integral, to divide the weight function appropriately amongst the three terms.

$\triangleright$ The case $\beta=2$. As described above, we let $\xi \equiv \xi_{\beta}=\int_{-\infty}^{x} \eta_{\beta}(z, t) d z$ for $\eta_{\beta} \in W_{\sigma, L-2,1}$. We have two cases to consider: $\alpha=(2,0)$ or $\alpha=(0,1)$.

$\triangleright$ The subcase $\alpha=(2,0)$. For the case $\alpha=(2,0)$, our remainder term satisfies

$$
\begin{aligned}
\left|\int_{0}^{t} \int \xi u_{x x}\left(u u_{x}\right)_{x x}\right| & =\left|\int_{0}^{t} \int \xi\left\{3 u_{x} u_{x x}+u u_{x x x}\right\} u_{x x}\right| \\
& \leq C\left|\int_{0}^{t} \int \xi u_{x} u_{x x}^{2}\right|+C\left|\int_{0}^{t} \int[\xi u]_{x} u_{x x}^{2}\right| \\
& \leq C\left|u_{x}\right|_{L^{\infty}}\left(\int_{0}^{t} \int \xi u_{x x}^{2}\right)+\left|\int_{0}^{t} \int\left(\xi_{x} u+\xi u_{x}\right) u_{x x}^{2}\right| \\
& \leq C\left(|u|_{L^{\infty}}+\left|u_{x}\right|_{L^{\infty}}\right)\left(\int_{0}^{t} \int \xi u_{x x}^{2}\right) \\
& \leq C\left(\int_{0}^{T} \int \xi u_{x x}^{2}\right),
\end{aligned}
$$

where $C$ depends only on $\|u\|_{X^{0}}$, using (2.12). Combining this bound on the remainder term with (2.14), for the case $\alpha=(2,0)$, we conclude,

$$
\sup _{0 \leq t \leq T} \int \xi u_{x x}^{2}+\int_{0}^{T} \int \eta u_{x x x}^{2}+\int_{0}^{T} \int \eta u_{x y}^{2} \leq C,
$$

where $C$ depends only on $\|u\|_{X^{0}}$ and (2.9)-(2.11), as desired.

$\triangleright$ The subcase $\alpha=(0,1)$. Similarly, for the case $\alpha=(0,1)$, our remainder term is bounded as follows

$$
\begin{aligned}
\left|\int_{0}^{t} \int \xi u_{y}\left(u u_{x}\right)_{y}\right| & =\left|\int_{0}^{t} \int \xi\left\{u_{y} u_{x}+u u_{x y}\right\} u_{y}\right| \\
& \leq C\left(|u|_{L^{\infty}}+\left|u_{x}\right|_{L^{\infty}}\right)\left(\int_{0}^{t} \int \xi u_{y}^{2}\right) \\
& \leq C\left(\int_{0}^{T} \int \xi u_{y}^{2}\right),
\end{aligned}
$$


where $C$ depends only on $\|u\|_{X^{0}}$, and, therefore,

$$
\sup _{0 \leq t \leq T} \int \xi u_{y}^{2}+\int_{0}^{T} \int \eta u_{x y}^{2}+\int_{0}^{T} \int \eta\left(\partial_{x}^{-1} u_{y y}\right)^{2} \leq C .
$$

$\triangleright$ The case $\beta=3$. We now take $\xi \equiv \xi_{\beta}=\int_{-\infty}^{x} \eta_{\beta}(z, t) d z$, for $\eta_{\beta} \in$ $W_{\sigma, L-3,2}$ and look at our two possibilities: $\alpha=(3,0)$ and $\alpha=(1,1)$.

$\triangleright$ The subcase $\alpha=(3,0)$. First, for the case $\alpha=(3,0)$, our remainder terms are,

$$
\left|\int_{0}^{t} \int \xi u_{x x x}\left(u u_{x}\right)_{x x x}\right|=\left|\int_{0}^{t} \int \xi\left\{3 u_{x x}^{2}+u_{x} u_{x x x}+u u_{x x x x}\right\} u_{x x x}\right| .
$$

We will look at these three terms separately. For many of these bounds, we will use estimate (2.12).

For our first term, $\int_{0}^{t} \int \xi u_{x x}^{2} u_{x x x}$, we will look at the cases $x>1$ and $x<-1$ separately. Let $A=\{x>1\} \times \mathbb{R}$ and $B=\{x<-1\} \times \mathbb{R}$. First, for $x>1$,

$$
\begin{aligned}
& \left|\int_{0}^{t} \int_{A} \xi u_{x x}^{2} u_{x x x}\right| \\
\leq & C\left|\int_{0}^{t} \int_{A} t^{2} x^{L-2} u_{x x}^{2} u_{x x x}\right| \\
\leq & C \int_{0}^{T}\left\{\left|t x^{(L-3) / 2} u_{x x}\right|_{L^{\infty}(A)}\left(\int_{A} t x^{L-1} u_{x x}^{2}\right)^{1 / 2}\left(\int_{A} t u_{x x x}^{2}\right)^{1 / 2}\right\} \\
\leq & \left(\sup _{0 \leq t \leq T} \int_{A} t x^{L-1} u_{x x}^{2}\right)^{1 / 2}\left(\int_{0}^{T}\left|t x^{(L-3) / 2} u_{x x}\right|_{L^{\infty}(A)}^{2}\right)^{1 / 2}\left(\int_{0}^{T} \int_{A} t u_{x x x}^{2}\right)^{1 / 2} \\
\leq & \frac{C}{\varepsilon}\left(\sup _{0 \leq t \leq T} \int_{A} t x^{L-1} u_{x x}^{2}\right)\left(\int_{0}^{T} \int_{A} t u_{x x x}^{2}\right)+\varepsilon\left(\int_{0}^{T}\left|t x^{(L-3) / 2} u_{x x}\right|_{L^{\infty}(A)}^{2}\right) \\
\leq & C+\varepsilon \int_{0}^{T} \int_{A} t^{2} x^{L-3}\left(u_{x x}^{2}+u_{x x x x}^{2}+u_{x x y}^{2}\right) \\
\leq & C+\varepsilon \int_{0}^{T} \int_{A} \xi_{x}\left(u_{x x x x}^{2}+u_{x x y}^{2}\right),
\end{aligned}
$$

while for $x<-1$, we can use the following estimate:

$$
\begin{aligned}
& \left|\int_{0}^{t} \int_{B} \xi u_{x x}^{2} u_{x x x}\right| \leq C\left|\int_{0}^{t} \int_{B} t^{2} e^{\sigma x} u_{x x}^{2} u_{x x x}\right| \\
& \quad \leq \int_{0}^{T}\left\{\left|t e^{\sigma x / 2} u_{x x}\right|_{L^{\infty}(B)}\left(\int_{B} t e^{\sigma x / 2} u_{x x}^{2}\right)^{1 / 2}\left(\int_{B} t e^{\sigma x / 2} u_{x x x}^{2}\right)^{1 / 2}\right\} \leq
\end{aligned}
$$




$$
\begin{aligned}
& \leq\left(\sup _{0 \leq t \leq T} \int_{B} t e^{\sigma x / 2} u_{x x}^{2}\right)^{1 / 2}\left(\int_{0}^{T}\left|t e^{\sigma x / 2} u_{x x}\right|_{L^{\infty}(B)}^{2}\right)^{1 / 2}\left(\int_{0}^{T} \int_{B} t e^{\sigma x / 2} u_{x x x}^{2}\right)^{1 / 2} \\
& \leq \frac{C}{\varepsilon}\left(\sup _{0 \leq t \leq T} \int_{B} t e^{\sigma x / 2} u_{x x}^{2}\right)\left(\int_{0}^{T} \int_{B} t e^{\sigma x / 2} u_{x x x}^{2}\right)+\varepsilon\left(\int_{0}^{T}\left|t e^{\sigma x / 2} u_{x x}\right|_{L^{\infty}(B)}^{2}\right) \\
& \leq C+\varepsilon \int_{0}^{T} \int_{B} t^{2} e^{\sigma x}\left(u_{x x}^{2}+u_{x x x x}^{2}+u_{x x y}^{2}\right) \\
& \leq C+\varepsilon \int_{0}^{T} \int_{B} \xi_{x}\left(u_{x x x x}^{2}+u_{x x y}^{2}\right) .
\end{aligned}
$$

Lastly, we have

$$
\begin{aligned}
\left|\int_{0}^{t} \int \xi u u_{x x x} u_{x x x x}\right| & =C\left|\int_{0}^{t} \int[\xi u]_{x} u_{x x x}^{2}\right| \\
& \leq C\left(|u|_{L^{\infty}}+\left|u_{x}\right|_{L^{\infty}}\right)\left(\int_{0}^{T} \int \xi u_{x x x}^{2}\right) \\
& \leq C\|u\|_{X^{0}}\left(\int_{0}^{T} \int \xi u_{x x x}^{2}\right) .
\end{aligned}
$$

Combining these estimates with $(2.14)$ in the case $\alpha=(3,0)$, we have $\sup _{0 \leq t \leq T} \int \xi u_{x x x}^{2}+3 \int_{0}^{T} \int \xi_{x} u_{x x x x}^{2}+\int_{0}^{T} \int \xi_{x} u_{x x y}^{2} \leq C+\varepsilon \int_{0}^{T} \int \xi_{x}\left(u_{x x x x}^{2}+u_{x x y}^{2}\right)$.

Bringing the $\varepsilon$ term to the left-hand side, we conclude

$$
\sup _{0 \leq t \leq T} \int \xi u_{x x x}^{2}+\int_{0}^{T} \int \eta u_{x x x x}^{2}+\int_{0}^{T} \int \eta u_{x x y}^{2} \leq C,
$$

where $C$ depends only on $\|u\|_{X^{0}}$ and (2.9)-(2.11), as desired.

$\triangleright$ The subcase $\alpha=(1,1)$. For the case $\alpha=(1,1)$, our remainder terms are

$$
\left|\int_{0}^{t} \int \xi u_{x y}\left(u u_{x}\right)_{x y}\right|=\left|\int_{0}^{t} \int \xi\left\{2 u_{x} u_{x y}+u_{y} u_{x x}+u u_{x x y}\right\} u_{x y}\right| .
$$

We will look at each of these terms below. For our first term,

$$
\left|\int_{0}^{t} \int \xi u_{x} u_{x y}^{2}\right| \leq\left|u_{x}\right|_{L^{\infty}}\left(\int_{0}^{T} \int \xi u_{x y}^{2}\right) .
$$


For the second term, we will look at the cases $x>1$ and $x<-1$ separately. We will again use the notation, $A=\{x>1\} \times \mathbb{R}, B=\{x<-1\} \times \mathbb{R}$. For $x>1$,

$$
\begin{aligned}
& \left|\int_{0}^{t} \int_{A} \xi u_{y} u_{x x} u_{x y}\right| \\
& \quad \leq C\left|\int_{0}^{t} \int_{A} t^{2} x^{L-2} u_{y} u_{x x} u_{x y}\right| \\
& \quad \leq C \int_{0}^{T}\left\{\left|t x^{(L-3) / 2} u_{y}\right|_{L^{\infty}(A)}\left(\int_{A} t x^{L-1} u_{x x}^{2}\right)^{1 / 2}\left(\int_{A} t u_{x y}^{2}\right)^{1 / 2}\right\} \\
& \quad \leq\left(\sup _{0 \leq t \leq T} \int_{A} t x^{L-1} u_{x x}^{2}\right)^{1 / 2}\left(\int_{0}^{T}\left|t x^{(L-3) / 2} u_{y}\right|_{L^{\infty}(A)}^{2}\right)^{1 / 2}\left(\int_{0}^{T} \int_{A} t u_{x y}^{2}\right)^{1 / 2} \\
& \quad \leq \frac{C}{\varepsilon}\left(\sup _{0 \leq t \leq T} \int_{A} t x^{L-1} u_{x x}^{2}\right)\left(\int_{0}^{T} \int_{A} t u_{x y}^{2}\right)+\varepsilon\left(\int_{0}^{T}\left|t x^{(L-3) / 2} u_{y}\right|_{L^{\infty}(A)}^{2}\right) \\
& \quad \leq C+\varepsilon \int_{0}^{T} \int_{A} t^{2} x^{L-3}\left(u_{y}^{2}+u_{x x y}^{2}+u_{y y}^{2}\right) \\
& \quad \leq C+\varepsilon \int_{0}^{T} \int_{A} \xi_{x}\left(u_{x x y}^{2}+u_{y y}^{2}\right),
\end{aligned}
$$

while for $x<-1$, we have:

$$
\begin{aligned}
& \left|\int_{0}^{t} \int_{B} \xi u_{y} u_{x x} u_{x y}\right| \\
& \leq C\left|\int_{0}^{t} \int_{B} t^{2} e^{\sigma x} u_{y} u_{x x} u_{x y}\right| \\
& \leq \int_{0}^{T}\left\{\left|t e^{\sigma x / 2} u_{y}\right|_{L^{\infty}(B)}\left(\int_{B} t e^{\sigma x / 2} u_{x x}^{2}\right)^{1 / 2}\left(\int_{B} t e^{\sigma x / 2} u_{x y}^{2}\right)^{1 / 2}\right\} \\
& \leq\left(\sup _{0 \leq t \leq T} \int_{B} t e^{\sigma x / 2} u_{x x}^{2}\right)^{1 / 2}\left(\int_{0}^{T}\left|t e^{\sigma x / 2} u_{y}\right|_{L^{\infty}(B)}^{2}\right)^{1 / 2}\left(\int_{0}^{T} \int_{B} t e^{\sigma x / 2} u_{x y}^{2}\right)^{1 / 2} \\
& \leq \frac{C}{\varepsilon}\left(\sup _{0 \leq t \leq T} \int_{B} t e^{\sigma x / 2} u_{x x}^{2}\right)\left(\int_{0}^{T} \int_{B} t e^{\sigma x / 2} u_{x y}^{2}\right)+\varepsilon\left(\int_{0}^{T}\left|t e^{\sigma x / 2} u_{y}\right|_{L^{\infty}(B)}^{2}\right) \\
& \leq C+\varepsilon \int_{0}^{T} \int_{B} t^{2} e^{\sigma x}\left(u_{y}^{2}+u_{x x y}^{2}+u_{y y}^{2}\right) \\
& \leq C+\varepsilon \int_{0}^{T} \int_{B} \xi_{x}\left(u_{x x y}^{2}+u_{y y}^{2}\right) .
\end{aligned}
$$

last term is handled as follows

$$
\left|\int_{0}^{t} \int \xi u u_{x x y} u_{x y}\right|=C\left|\int_{0}^{t} \int[\xi u]_{x} u_{x y}^{2}\right| \leq C\left(|u|_{L^{\infty}}+\left|u_{x}\right|_{L^{\infty}}\right)\left(\int_{0}^{T} \int \xi u_{x y}^{2}\right) .
$$


Combining these estimates with (2.14) for the case $\alpha=(1,1)$, we have

$$
\sup _{0 \leq t \leq T} \int \xi u_{x y}^{2}+\int_{0}^{T} \int \xi_{x} u_{x x y}^{2}+\int_{0}^{T} \int \xi_{x} u_{y y}^{2} \leq C+\varepsilon \int_{0}^{T} \int \xi_{x}\left(u_{x x y}^{2}+u_{y y}^{2}\right) \text {. }
$$

Bringing the $\varepsilon$ term on to the left-hand side, we get

$$
\sup _{0 \leq t \leq T} \int \xi u_{x y}^{2}+\int_{0}^{T} \int \eta u_{x x y}^{2}+\int_{0}^{T} \int \eta u_{y y}^{2} \leq C,
$$

where $C$ depends only on $\|u\|_{X^{0}}$ and (2.9)-(2.11).

$\triangleright$ The case $\beta \geq 4$. For $\beta \geq 4$, we let $\xi \equiv \xi_{\beta}$ be defined as in (2.13). We combine Lemma 2.5 given below, in which we estimate our remainder term (2.15), with our main inequality (2.14) to conclude that

$$
\sup _{0 \leq t \leq T} \int \xi\left(\partial^{\alpha} u\right)^{2}+\int_{0}^{T} \int \eta\left(\partial^{\alpha} u_{x}\right)^{2}+\int_{0}^{T} \int \eta\left(\partial^{\alpha}\left(\partial_{x}^{-1} u_{y}\right)\right)^{2} \leq C .
$$

Thus, our lemma is proved.

We now show the bounds on the remainder (2.15) for $\beta \geq 4$.

Lemma 2.5. (Estimates of the remainder) For $4 \leq \beta \leq L+1, \beta=$ $\alpha_{1}+2 \alpha_{2},\left(\alpha_{1}, \alpha_{2}\right) \in \mathbb{Z}^{+} \times \mathbb{Z}^{+}$, and $u$ a solution of $(2.1)$ sufficiently smooth and with sufficient decay at infinity, for $0 \leq t \leq T$, we have

$$
\left|\int_{0}^{t} \int \xi_{\beta}\left(\partial^{\alpha} u\right) \partial^{\alpha}\left(u u_{x}\right)\right| \leq C
$$

where $\xi_{\beta} \in W_{\sigma, L-(\beta-1)^{+},(\beta-1)^{+}}, \eta_{\beta} \in W_{\sigma, L-(\beta-1)^{+}-1,(\beta-1)^{+}}, \sigma>0$ arbitrary, and $C$ depends only on $\|u\|_{X^{0}}$ and on (2.9)-(2.11).

Before proving these estimates, we describe the form of each term in (2.16).

Lemma 2.6. (Form of remainder terms) Let $\alpha=\left(\alpha_{1}, \alpha_{2}\right) \in \mathbb{Z}^{+} \times \mathbb{Z}^{+}$. Then every term in the integrand of

$$
\int_{0}^{t} \int \xi\left(\partial^{\alpha} u\right) \partial^{\alpha}\left(u u_{x}\right)
$$

is of the form

$$
C \xi\left(\partial^{\alpha} u\right)\left(\partial^{r} u\right)\left(\partial^{s} u_{x}\right)
$$

where $r=\left(r_{1}, r_{2}\right), s=\left(s_{1}, s_{2}\right), r, s \in \mathbb{Z}^{+} \times \mathbb{Z}^{+}, r_{i}+s_{i}=\alpha_{i}$ for $i=1,2$.

Proof. The proof follows by applying Leibniz' formula to $\partial^{\alpha}\left(u u_{x}\right)$. 
We now turn to the proof of Lemma 2.5.

\section{Proof of Lemma 2.5.}

By Lemma 2.6, we can write every term of

$$
\int_{0}^{t} \int \xi_{\beta}\left(\partial^{\alpha} u\right) \partial^{\alpha}\left(u u_{x}\right)
$$

in the form

$$
\int_{0}^{t} \int \xi\left(\partial^{\alpha} u\right)\left(\partial^{r} u\right)\left(\partial^{s} u_{x}\right)
$$

where $\xi \equiv \xi_{\beta} \in W_{\sigma, L-(\beta-1)^{+},(\beta-1)^{+}}$is defined as in (2.13). It remains to show that each of these terms is bounded by constants depending only on (2.9)-(2.11).

In particular, we will focus on the case when $x>1$. Again, we will let $A=\{x>1\} \times \mathbb{R}$. In $A$ our weight function $\xi \approx t^{k} x^{\ell}$ for $k, \ell \geq 0$. When $x<-1, \xi \approx t^{k} e^{\sigma x}$ for $\sigma>0$ arbitrary. This case can be handled even easier. In particular, therefore, for $x>1$, we are looking to get bounds on (2.19) in terms of:

$$
\begin{aligned}
& \sup _{0 \leq t \leq T} \int_{A} t^{(v-1)^{+}} x^{L-(v-1)^{+}}\left(\partial^{\gamma} u\right)^{2}, \\
& \int_{0}^{T} \int_{A} t^{(v-1)^{+}} x^{L-(v-1)^{+}-1}\left(\partial^{\gamma} u_{x}\right)^{2}, \\
& \int_{0}^{T} \int_{A} t^{(v-1)^{+}} x^{L-(v-1)^{+}-1}\left(\partial^{\gamma}\left(\partial_{x}^{-1} u_{y}\right)\right)^{2},
\end{aligned}
$$

where $\beta-1 \geq v=\gamma_{1}+2 \gamma_{2} \geq 0$. We need to break up each term of the form (2.19) into three parts, being sure to divide the weight function appropriately among the three terms.

For example, we would like to estimate $\left|\omega \partial^{\gamma} u\right|_{L^{\infty}}$ where $\omega$ is part of our weight function $\xi$. In order to do so, we combine the following imbedding (see (2.12))

$$
\left|\partial^{\gamma} u\right|_{L^{\infty}} \leq\left(\int\left(\partial^{\gamma} u\right)^{2}+\left(\partial^{\gamma} u_{x x}\right)^{2}+\left(\partial^{\gamma} u_{y}\right)^{2}\right)^{1 / 2},
$$

with the fact that

$$
t^{k} x^{\ell}\left(\partial^{\alpha} u\right)=t^{k} \sum_{j=0}^{\alpha_{1}}(-1)^{j+1}\left(\begin{array}{c}
\alpha \\
j
\end{array}\right) \partial_{x}^{j}\left\{\left(\partial_{x}^{\alpha_{1}-j} x^{\ell}\right)\left(\partial_{y}^{\alpha_{2}} u\right)\right\} .
$$


First, we know that

$$
\left|t^{k} x^{\ell}\left(\partial^{\gamma} u\right)\right|_{L^{\infty}} \leq t^{k} \sum_{j=0}^{\alpha_{1}}\left|\left(\begin{array}{c}
\alpha \\
j
\end{array}\right) \partial_{x}^{j}\left\{\left(\partial_{x}^{\alpha_{1}-j} x^{\ell}\right)\left(\partial_{y}^{\alpha_{2}} u\right)\right\}\right|_{L^{\infty}}
$$

Therefore, we combine (2.24) with (2.23) to conclude that

(2.25) $\sup _{0 \leq t \leq T}\left|t^{(v+1) / 2} x^{(L-(v+1)) / 2}\left(\partial^{\gamma} u\right)\right|_{L^{\infty}(A)} \leq C \quad$ for $v=\gamma_{1}+2 \gamma_{2} \leq \beta-3$, (2.26) $\int_{0}^{T}\left|t^{v / 2} x^{(L-(v+1)) / 2}\left(\partial^{\gamma} u\right)\right|_{L^{\infty}(A)}^{2} d t \leq C \quad$ for $v=\gamma_{1}+2 \gamma_{2} \leq \beta-2$, where the constants $C$ depend only on (2.20)-(2.22).

With these inequalities in mind, we look at terms of the form (2.19). For $x>1$, these terms can be expressed as follows

$$
\left|\int_{0}^{t} \int_{A} \xi\left(\partial^{\alpha} u\right)\left(\partial^{r} u\right)\left(\partial^{s} u_{x}\right)\right| \leq C \int_{0}^{T}\left|\int_{A} t^{\beta-1} x^{L-(\beta-1)}\left(\partial^{\alpha} u\right)\left(\partial^{r} u\right)\left(\partial^{s} u_{x}\right)\right| .
$$

For notation, let $v_{r}=r_{1}+2 r_{2}$ and $v_{s}=s_{1}+2 s_{2}$. Recall that $\beta=\alpha_{1}+2 \alpha_{2}$, and therefore $v_{r}+v_{s}=\beta$.

$\triangleright$ The case $v_{s} \leq \beta-4$. In this case, we can bound the remainder term as follows

$$
\begin{aligned}
\int_{0}^{T}\left|\int_{A} t^{\beta-1} x^{L-(\beta-1)}\left(\partial^{\alpha} u\right)\left(\partial^{r} u\right)\left(\partial^{s} u_{x}\right)\right| \\
\leq \sup _{x>1}\left(x^{N} T^{M}\right) \sup _{0 \leq t \leq T}\left|t^{\left(v_{s}+2\right) / 2} x^{\left(L-\left(v_{s}+2\right)\right) / 2}\left(\partial^{s} u_{x}\right)\right|_{L^{\infty}(A)} \\
\cdot\left(\int_{0}^{T} \int_{A} t^{\left(v_{r}-2\right)^{+}} x^{L-\left(v_{r}-2\right)^{+}-1}\left(\partial^{r} u\right)^{2}\right)^{1 / 2} \\
\cdot\left(\int_{0}^{T} \int_{A} t^{\beta-2} x^{L-(\beta-2)-1}\left(\partial^{\alpha} u\right)^{2}\right)^{1 / 2}
\end{aligned}
$$

First, we will show that $M \geq 0$ and $N \leq 0$, so that any extra powers of $t$ or $x$ can be thrown away.

$$
M=(\beta-1)-\left(\frac{\nu_{s}+2}{2}\right)-\left(\frac{\left(\nu_{r}-2\right)^{+}}{2}\right)-\left(\frac{\beta-2}{2}\right) .
$$

Therefore, $2 M=\beta-v_{s}-2-\left(v_{r}-2\right)^{+} \geq \beta-v_{s}-2-v_{r}+2=0$, while $N=(L-(\beta-1))-\left(\frac{L-\left(\nu_{s}+2\right)}{2}\right)-\left(\frac{L-\left(v_{r}-2\right)^{+}-1}{2}\right)-\left(\frac{L-(\beta-2)-1}{2}\right)$ 
implies $2 N=-L-\beta+4+v_{s}+\left(v_{r}-2\right)^{+} \leq-L-\beta+4+v_{s}+v_{r}-2=-L+2 \leq 0$ by the assumption that $L \geq 2$. The other three terms are bounded by (2.25), (2.21), and (2.22).

$\triangleright$ The case $\beta-4<v_{s} \leq \beta-2$. First, if $\beta \geq 5$, then $v_{r} \leq 3 \leq \beta-2$. This fact will allow us to use estimate (2.26) on the term involving $\left(\partial^{r} u\right)$. In addition, if $\beta=4$ and $v_{s}=\beta-2$, then $v_{r}=2 \leq \beta-2$, which will allow us to use the same estimate on the $\left(\partial^{r} u\right)$ term. For these cases, we estimate as follows

$$
\begin{gathered}
\int_{0}^{T}\left|\int_{A} t^{\beta-1} x^{L-(\beta-1)}\left(\partial^{\alpha} u\right)\left(\partial^{r} u\right)\left(\partial^{s} u_{x}\right)\right| \\
\leq C \sup _{x>1}\left(x^{N} T^{M}\right)\left(\sup _{0 \leq t \leq T} \int_{A} t^{v_{s}} x^{L-v_{s}}\left(\partial^{s} u_{x}\right)^{2}\right)^{1 / 2} \\
\cdot\left(\int_{0}^{T}\left|t^{v_{r} / 2} x^{\left(L-\left(v_{r}+1\right)\right) / 2}\left(\partial^{r} u\right)\right|_{L^{\infty}(A)}^{2}\right)^{1 / 2} \\
\cdot\left(\int_{0}^{T} \int_{A} t^{\beta-2} x^{L-(\beta-2)-1}\left(\partial^{\alpha} u\right)^{2}\right)^{1 / 2}
\end{gathered}
$$

First, we know

$$
M=(\beta-1)-\left(\frac{\nu_{s}}{2}\right)-\left(\frac{\nu_{r}}{2}\right)-\left(\frac{\beta-2}{2}\right)
$$

implies $2 M=2 \beta-2 v_{s}-v_{r}-\beta+2=0$, while

$$
N=(L-(\beta-1))-\left(\frac{L-v_{s}}{2}\right)-\left(\frac{L-\left(\nu_{r}+1\right)}{2}\right)-\left(\frac{L-(\beta-2)-1}{2}\right)
$$

implies $2 N=-L+2 \leq 0$ for $L \geq 2$, so we can throw away any extra powers of $x$. The other three terms are bounded as follows. By assumption, $v_{s} \leq \beta-2$. Therefore, the first term is bounded by (2.20). The second term is bounded by (2.26) because $v_{r} \leq \beta-2$. Clearly, the third term is bounded by (2.21) or (2.22) as desired. If $\beta=4$ and $v_{s}=\beta-3$, on the other hand, we can use the following estimate

$$
\begin{aligned}
& \int_{0}^{T}\left|\int_{A} t^{\beta-1} x^{L-(\beta-1)}\left(\partial^{\alpha} u\right)\left(\partial^{r} u\right)\left(\partial^{s} u_{x}\right)\right| \\
& \quad=\int_{0}^{T}\left|\int_{A} t^{\beta-1} x^{L-(\beta-1)}\left(\partial^{\alpha} u\right) u_{x}\left(\partial^{\alpha} u\right)\right| \\
& \quad \leq C T\|u\|_{X^{0}}\left(\int_{0}^{T} \int_{A} t^{\beta-2} x^{L-(\beta-1)}\left(\partial^{\alpha} u\right)^{2}\right)^{1 / 2},
\end{aligned}
$$

which is clearly bounded by (2.21) or (2.22), as desired. 
$\triangleright$ The case $v_{s}=\beta-1$. This case is handled in the same manner as the case $\beta=4, v_{s}=\beta-3$ above.

The case $v_{s}=\beta$. In this case $v_{r}=0$, and therefore

$$
\begin{aligned}
& \int_{0}^{T}\left|\int_{A} t^{\beta-1} x^{L-(\beta-1)}\left(\partial^{\alpha} u\right)\left(\partial^{r} u\right)\left(\partial^{s} u_{x}\right)\right| \\
& \quad=\int_{0}^{T}\left|\int_{A} t^{\beta-1} x^{L-(\beta-1)} u\left(\partial^{\alpha} u\right)\left(\partial^{\alpha} u_{x}\right)\right| \\
& \quad=C \int_{0}^{T}\left|\int_{A} t^{\beta-1}\left[x^{L-(\beta-1)} u\right]_{x}\left(\partial^{\alpha} u\right)^{2}\right| \\
& \quad \leq C T\left(|u|_{L^{\infty}}+\left|u_{x}\right|_{L^{\infty}}\right)\left(\int_{0}^{T} \int_{A} t^{\beta-2} x^{L-(\beta-1)}\left(\partial^{\alpha} u\right)^{2}\right) \\
& \quad \leq C T\|u\|_{X^{0}}\left(\int_{0}^{T} \int_{A} t^{\beta-2} x^{L-(\beta-1)}\left(\partial^{\alpha} u_{x}\right)^{2}\right),
\end{aligned}
$$

where again the right-hand side is bounded by (2.21) or (2.22). Thus, our lemma is proved.

3. EXISTENCE AND UNIQUENESS. In this section we will prove that, for $\varphi \in X^{N}\left(\mathbb{R}^{2}\right)$, there exists a unique solution $u$ of (2.1) in $L^{\infty}\left([0, T] ; X^{N}\left(\mathbb{R}^{2}\right)\right)$, where the time of existence $T$ depends only on $\|\varphi\|_{X^{0}}$.

Theorem 3.1. (Uniqueness) For $\varphi \in X^{0}\left(\mathbb{R}^{2}\right)$ there is at most one solution of $(2.1)$ in $L^{\infty}\left([0, T] ; X^{0}\left(\mathbb{R}^{2}\right)\right)$.

Proof. Assume there are two solutions of $(2.1)$ in $L^{\infty}\left([0, T] ; X^{0}\left(\mathbb{R}^{2}\right)\right)$ with the same initial data. By equation $(2.1), \partial_{t} u, \partial_{t} v \in L^{\infty}\left([0, T] ; L^{2}\left(\mathbb{R}^{2}\right)\right)$, so all integrations below are justified. We begin by multiplying $(2.1)$ by $2(u-v)$ and integrating over $\mathbb{R}^{2}$. Therefore,

$$
\begin{aligned}
2 \int\left[(u-v)(u-v)_{t}+(u-v)(u-v)_{x x x}+(u-v)(u-v)_{x}\right. \\
\left.+(u-v) \partial_{x}^{-1}(u-v)_{y y}+(u-v)\left(u u_{x}-v v_{x}\right)\right]=0
\end{aligned}
$$

After integrating by parts, we have:

$$
\partial_{t} \int(u-v)^{2}+\int(u-v)\left(u u_{x}-v v_{x}\right)=0,
$$


which implies

$$
\partial_{t} \int(u-v)^{2}+\int(u-v)\left(u u_{x}-u v_{x}+u v_{x}-v v_{x}\right)=0,
$$

and thus

$$
\begin{aligned}
\partial_{t} \int(u-v)^{2} & \leq C\left|\int(u-v)\left(u u_{x}-u v_{x}\right)+(u-v)\left(u v_{x}-v v_{x}\right)\right| \\
& \leq C\left|\int(u-v)^{2} u_{x}\right|+C\left|\int(u-v)^{2} v_{x}\right| \\
& \leq C\left(\left|u_{x}\right|_{L^{\infty}}+\left|v_{x}\right|_{L^{\infty}}\right) \int(u-v)^{2} \\
& \leq C\left(\|u\|_{X^{0}}+\|v\|_{X^{0}}\right) \int(u-v)^{2} .
\end{aligned}
$$

Consequently, using Gronwall's inequality and the fact that $u(\cdot, 0)=v(\cdot, 0)$, we have that $u \equiv v$.

We would now like to prove a local existence theorem for (2.1). Our plan is to show that for $\varphi \in X^{N}\left(\mathbb{R}^{2}\right)$ there exists a solution $u \in$ $L^{\infty}\left([0, T] ; X^{N}\left(\mathbb{R}^{2}\right)\right)$ for a time $T$ depending only on $\|\varphi\|_{X^{0}}$. We will do so as follows. First we will introduce the following spaces. Let

$$
Y^{N}\left(\mathbb{R}^{2}\right) \equiv\left\{f \mid f, f_{x x x}, f_{y y}, \frac{\eta^{2}}{\xi} \hat{f} \in H^{N}\left(\mathbb{R}^{2}\right)\right\},
$$

with the accompanying norm

$$
\|f\|_{Y^{N}}^{2} \equiv \int_{\mathbb{R}^{2}} f^{2}+\sum_{|\alpha| \leq N}\left\{\left(\partial^{\alpha} f_{x x x}\right)^{2}+\left(\partial_{x}^{-1}\left(\partial^{\alpha} f_{y y}\right)\right)^{2}+\left(\partial^{\alpha} f_{y y}\right)^{2}\right\} .
$$

In addition, let

$$
Z_{T}^{N} \equiv\left\{f \mid f \in L^{\infty}\left([0, T] ; H^{(N+3, N+2)}\left(\mathbb{R}^{2}\right)\right), f_{t} \in L^{\infty}\left([0, T] ; H^{N}\left(\mathbb{R}^{2}\right)\right)\right\},
$$

where $H^{(k, m)}\left(\mathbb{R}^{2}\right) \equiv\left\{f \mid f, \partial_{x}^{k} f, \partial_{y}^{m} f \in L^{2}\left(\mathbb{R}^{2}\right)\right\}$, with the accompanying norm

$\|f\|_{Z_{T}^{N}}^{2} \equiv \sup _{0 \leq t \leq T} \int_{\mathbb{R}^{2}} f^{2}+\sum_{|\alpha|=N}\left\{\left(\partial^{\alpha} f_{x x x}\right)^{2}+\left(\partial^{\alpha} f_{y y}\right)^{2}\right\}+\int_{\mathbb{R}^{2}} f_{t}^{2}+\sum_{|\alpha|=N}\left(\partial^{\alpha} f_{t}\right)^{2}$.

We will begin by showing that, for $\varphi \in Y^{N}\left(\mathbb{R}^{2}\right)$, there exists a solution $u$ of (2.1) such that $u \in L^{\infty}\left([0, T] ; Y^{N}\left(\mathbb{R}^{2}\right)\right)$ for a time $T$ depending only on $\|\varphi\|_{Y^{0}}$. Then we will prove a differential inequality of the form

$$
\partial_{t}\left(\int u^{2}+u_{x x x}^{2}+\left(\partial_{x}^{-1} u_{y y}\right)^{2}\right) \leq\left(\int u^{2}+u_{x x x}^{2}+\left(\partial_{x}^{-1} u_{y y}\right)^{2}\right)^{3 / 2}
$$


in order to show that in fact the solution $u$ obtained in Theorem 3.2 is in $L^{\infty}\left(\left[0, T^{\prime}\right] ; X^{0}\left(\mathbb{R}^{2}\right)\right)$ for a time $T^{\prime}$ depending only on $\|\varphi\|_{X^{0}\left(\mathbb{R}^{2}\right)}$. With these ideas in mind, we state our existence theorem.

Theorem 3.2. (Existence) Let $k_{0}>0$ and let $N$ be an integer $\geq 0$. Then there exists a time $0<T<\infty$, depending only on $k_{0}$, such that, for all $\varphi \in Y^{N}\left(\mathbb{R}^{2}\right)$ with $\|\varphi\|_{Y^{0}\left(\mathbb{R}^{2}\right)} \leq k_{0}$, there exists a solution of equation (2.1), $u \in L^{\infty}\left([0, T] ; Y^{N}\left(\mathbb{R}^{2}\right)\right)$, such that $u(x, y, 0)=\varphi(x, y)$.

The method of proof is as follows. We begin by approximating (2.1) by a sequence of linear equations. We then show that the sequence of solutions to our linear equations is bounded in $L^{\infty}\left([0, T] ; Y^{0}\left(\mathbb{R}^{2}\right)\right)$ for a time $T$ depending only on $\|\varphi\|_{Y^{0}}$. Third, we prove that a subsequence of solutions to our approximate equations converges to a solution $u \in L^{\infty}\left([0, T] ; Y^{0}\left(\mathbb{R}^{2}\right)\right)$ of (2.1). Lastly, we show that, if $\varphi \in Y^{N}\left(\mathbb{R}^{2}\right)$ for $N>0$, then, in fact, our solution $u \in L^{\infty}\left([0, T] ; Y^{N}\left(\mathbb{R}^{2}\right)\right)$, where the time $T$ depends only on $\|\varphi\|_{Y^{0}}$.

Proof. It suffices to prove this result for $\varphi \in \bigcap_{k \geq 0} H^{k}\left(\mathbb{R}^{2}\right)$ and $\partial_{x}^{-1} \varphi_{y y} \in$ $\bigcap_{k \geq 0} H^{k}\left(\mathbb{R}^{2}\right)$. We can then use the same approximation procedure as before to prove the result for general initial data.

We begin by approximating (2.1) by the linear equation

$$
u_{t}^{(n)}+u_{x x x}^{(n)}+u_{x}^{(n)}+\partial_{x}^{-1} u_{y y}^{(n)}+u^{(n-1)} u_{x}^{(n)}=0,
$$

where the initial condition is given by $u^{(n)}(x, y, 0)=\varphi(x, y)$, and the first approximation is given by $u^{(0)}(x, y, t)=\varphi(x, y)$. We will show that for each $n$ there exists a unique solution $u^{(n)}$ such that $u^{(n)} \in Z_{T}^{N}$ for a time $T$ independent of $n$. We will then show a subsequence of $\left\{u^{(n)}\right\}$ converges to a solution $u$.

Equation (3.1) is a linear equation which, by Lemma 3.4 given below, can be solved at each iteration. In particular, for each $n$ there exists a unique solution $u^{(n)}$ and, by Lemma 3.3,

$$
\left\|u^{(n)}\right\|_{Z_{t}^{0}}^{2} \leq\left\|u^{(n)}(\cdot, 0)\right\|_{H^{3,2}}^{2}+\left\|u_{t}^{(n)}(\cdot, 0)\right\|_{L^{2}}^{2}+C t\left\|u^{(n-1)}\right\|{ }_{Z_{t}^{0}}\left\|u^{(n)}\right\|_{Z_{t}^{0}}^{2} .
$$

By assumption, $k_{0} \geq\|\varphi\|_{Y^{0}}$. Note that

$$
\begin{aligned}
& \left\|u^{(n)}(\cdot, 0)\right\|_{H^{3,2}}^{2}+\left\|u_{t}^{(n)}(\cdot, 0)\right\|_{L^{2}}^{2} \\
= & \left\|u^{(n)}(\cdot, 0)\right\|_{H^{3,2}}^{2}+\int\left\{u_{x x x}^{(n)}(\cdot, 0)+u_{x}^{(n)}(\cdot, 0)+\partial_{x}^{-1} u_{y y}^{(n)}(\cdot, 0)+u^{(n-1)} u_{x}^{(n)}(\cdot, 0)\right\}^{2} \\
\leq & \|\varphi\|_{Y^{0}}^{2}+C \int\left\{\varphi_{x x x}^{2}+\varphi_{x}^{2}+\left(\partial_{x}^{-1} \varphi_{y y}\right)^{2}+\left(\varphi \varphi_{x}\right)^{2}\right\} \leq K\|\varphi\|_{Y^{0}}^{2} \leq K k_{0}^{2},
\end{aligned}
$$


where $K$ is clearly independent of $n$. Define $c_{0}^{2} \equiv K k_{0}^{2}+1$. Let

$$
T_{0}^{(n)} \equiv \sup \left\{t:\left\|u^{(j)}\right\|_{Z_{t}^{0}} \leq c_{0} \text { for } 0 \leq j \leq n\right\} .
$$

Therefore, for $t$ in the interval $\left[0, T_{0}^{(n)}\right]$,

$$
\begin{aligned}
\left\|u^{(n)}\right\|_{Z_{t}^{0}}^{2} & \leq\left\|u^{(n)}(\cdot, 0)\right\|_{H^{3,2}}^{2}+\left\|u_{t}^{(n)}(\cdot, 0)\right\|_{L^{2}}^{2}+C t\left\|u^{(n-1)}\right\|_{Z_{t}^{0}}\left\|u^{(n)}\right\|_{Z_{t}^{0}}^{2} \\
& \leq K k_{0}^{2}+C t c_{0}^{3} .
\end{aligned}
$$

Now, choose $T>0$ such that

$$
C T c_{0}^{3}=1 .
$$

We claim that $T_{0}^{(n)} \geq T$ for all $n$ and therefore, the sequence of approximate solutions $\left\{u^{(n)}\right\}$ is bounded for the time $T$, which is independent of $n$. If $T_{0}^{(n)}=\infty$, then clearly $T_{0}^{(n)} \geq T$. So, assume $T_{0}^{(n)}<\infty$. Suppose $T>$ $T_{0}^{(n)}$. Then, by the continuity of $\left\|u^{(n)}\right\|_{Z_{t}^{0}}$ with respect to $t$, we have $c_{0}^{2}=$ $\left\|u^{(j)}\right\|_{Z_{T_{0}^{(n)}}^{0}}^{2}$ for some integer $j \in[0, n]$. Therefore, by (3.2) above,

$$
c_{0}^{2}=\left\|u^{(j)}\right\|_{Z_{T_{0}^{(n)}}^{0}}^{2} \leq K k_{0}^{2}+C T_{0}^{(n)} c_{0}^{3}<K k_{0}^{2}+C T c_{0}^{3} \leq c_{0}^{2} .
$$

However, this implies $c_{0}^{2}<c_{0}^{2}$ and we have a contradiction. Thus, we conclude that $T_{0}^{(n)} \geq T$, and therefore

$$
\sup _{0 \leq t \leq T} \int\left(u^{(n)}(\cdot, t)\right)^{2}+\left(u_{x x x}^{(n)}(\cdot, t)\right)^{2}+\left(u_{y y}^{(n)}(\cdot, t)\right)^{2}+\left(u_{t}^{(n)}(\cdot, t)\right)^{2} \leq c_{0}^{2} .
$$

Consequently, there exists a bounded sequence of solutions $\left\{u^{(n)}\right\} \in$ $Z_{T}^{0}$, and therefore a subsequence, still denoted $\left\{u^{(n)}\right\}$, such that $u^{(n)}$ converges weak* to some $v \in L^{\infty}\left([0, T] ; H^{(3,2)}\left(\mathbb{R}^{2}\right)\right)$ and $u_{t}^{(n)}-v_{t}$ weak* in $L^{\infty}\left([0, T] ; L^{2}\left(\mathbb{R}^{2}\right)\right)$. Therefore, by Aubin's compactness theorem, $u^{(n)} \rightarrow v$ strongly in $L^{\infty}\left([0, T] ; H_{\text {loc }}^{1}\left(\mathbb{R}^{2}\right)\right)$. Now it remains to show that each term in (3.1) converges to its correct limit.

First, $u_{x x x}^{(n)}-v_{x x x}$ weak ${ }^{*}$ in $L^{\infty}\left([0, T] ; L^{2}\left(\mathbb{R}^{2}\right)\right)$. Similarly, $u_{t}^{(n)} \rightarrow v_{t}$ and $u_{x}^{(n)} \rightarrow v_{x}$ weak ${ }^{*}$ in $L^{\infty}\left([0, T] ; L^{2}\left(\mathbb{R}^{2}\right)\right)$.

Now we will show that the nonlinear term converges to its correct limit. First, $u^{(n-1)} \rightarrow v$ strongly in $L^{\infty}\left([0, T] ; H_{\mathrm{loc}}^{1}\left(\mathbb{R}^{2}\right)\right)$. Second, $u_{x}^{(n-1)} \rightarrow v_{x}$ 
weak* in $L^{\infty}\left([0, T] ; L^{2}\left(\mathbb{R}^{2}\right)\right)$. Therefore, $u^{(n-1)} u_{x}^{(n)} \rightarrow v v_{x}$ weak* in $L^{\infty}\left([0, T] ; L^{2}\left(\mathbb{R}^{2}\right)\right)$. Consequently,

$$
\begin{aligned}
\partial_{x}^{-1} u_{y y}^{(n)} & =-\left\{u_{t}^{(n)}+u_{x x x}^{(n)}+u_{x}^{(n)}+u^{(n-1)} u_{x}^{(n)}\right\} \\
& \rightarrow-\left\{v_{t}+v_{x x x}+v_{x}+v v_{x}\right\}
\end{aligned}
$$

weak* in $L^{\infty}\left([0, T] ; L^{2}\left(\mathbb{R}^{2}\right)\right)$. But, also note that $u_{y y}^{(n)} \rightarrow v_{y y}$ weak* in $L^{\infty}\left([0, T] ; L^{2}\left(\mathbb{R}^{2}\right)\right)$. Therefore, $\partial_{x}^{-1} u_{y y}^{(n)} \rightarrow \partial_{x}^{-1} v_{y y}$ weak* in $L^{\infty}\left([0, T] ; L^{2}\left(\mathbb{R}^{2}\right)\right)$ and consequently $v$ is a solution to (2.1).

Next we will prove that if $\varphi \in Y^{N}\left(\mathbb{R}^{2}\right)$ for some integer $N>0$, then the solution $u$ we just obtained is actually in $L^{\infty}\left([0, T] ; Y^{N}\left(\mathbb{R}^{2}\right)\right)$ for the time $T$ chosen in (3.3). We already know there is a solution $u \in L^{\infty}\left([0, T] ; Y^{0}\left(\mathbb{R}^{2}\right)\right)$. Therefore, we only need to show that the approximating sequence $\left\{u^{(n)}\right\}$ is bounded in $Z_{T}^{N}$ and, thus, by the convergence arguments above, our solution $u$ is in $L^{\infty}\left([0, T] ; Y^{N}\left(\mathbb{R}^{2}\right)\right)$. We use the same argument as before. By Lemma 3.4, we know our linearized equation can be solved in any interval of time in which the coefficients are defined. Therefore, for each iterate, $\left\|u^{(n)}\right\|_{Z_{t}^{N}}$ is continuous in $t \in[0, T]$. From Lemma 3.3, we know

$$
\left\|u^{(n)}\right\|_{Z_{t}^{N}}^{2} \leq\left\|u^{(n)}(\cdot, 0)\right\|_{H^{N+3, N+2}}^{2}+\left\|u_{t}^{(n)}(\cdot, 0)\right\|_{H^{N}}^{2}+C t\left\|u^{(n-1)}\right\|_{Z_{t}^{N}}\left\|u^{(n)}\right\|_{Z_{t}^{N}}^{2} .
$$

As before, we also know that

$$
\left\|u^{(n)}(\cdot, 0)\right\|_{H^{N+3, N+2}}^{2}+\left\|u_{t}^{(n)}(\cdot, 0)\right\|_{H^{N}}^{2} \leq K_{N}\|\varphi\|_{Y^{N}}^{2},
$$

where $K_{N}$ is independent of $n$. Let $c_{N}^{2} \equiv K_{N}\|\varphi\|_{Y^{N}}^{2}+1$. Let

$$
T_{N}^{(n)} \equiv \sup \left\{t:\left\|u^{(j)}\right\|_{Z_{t}^{N}} \leq c_{N} \text { for } 0 \leq j \leq n\right\} .
$$

Therefore, for $0 \leq t \leq T_{N}^{(n)}$,

$$
\left\|u^{(n)}\right\|_{Z_{t}^{N}}^{2} \leq K_{N}\|\varphi\|_{Y^{N}}^{2}+C t c_{N}^{3}
$$

Now choosing $T_{N}$ such that

$$
C T_{N} c_{N}^{3}=1,
$$

by the same arguments as in the case $N=0$, we conclude that $T_{N}^{(n)} \geq T_{N}$, and, therefore,

$$
\left\|u^{(n)}\right\|_{Z_{T^{N}}^{N}}^{2} \leq c_{N}^{2}
$$


Now, let

$$
T_{N}^{*} \equiv \sup \left\{t \mid u \in Z_{t}^{N}\right\} .
$$

We claim that $T_{N}^{*} \geq T$ and, therefore, a time of existence can be chosen depending only on $\|\varphi\|_{Y^{0}}$. By Lemma 3.4 the linear equation (3.1) can be solved in any interval of time in which the coefficients are defined, and, thus $T_{N}^{*} \geq T$.

We now prove the inequality used in our existence theorem above.

Lemma 3.3. Let $v, w$ be a pair of functions in $Z_{t}^{N}$ for all $N$ and all $t \geq 0$, such that $v, w$ are solutions to

$$
v_{t}+v_{x x x}+v_{x}+\partial_{x}^{-1} v_{y y}+w v_{x}=0
$$

Then for all $N \geq 0$, the following inequality holds:

$$
\|v\|_{Z_{t}^{N}}^{2} \leq\|v(\cdot, 0)\|_{H^{N+3, N+2}}^{2}+\left\|v_{t}(\cdot, 0)\right\|_{H^{N}}^{2}+C t\|w\|_{Z_{t}^{N}}\|v\|_{Z_{t}^{N}}^{2}
$$

for all $t \geq 0$.

Proof. We will show that for each $\alpha,|\alpha| \geq 0$ and $0 \leq \tilde{t} \leq t$,

$$
\begin{aligned}
& \partial_{t} \int\left(\partial^{\alpha} v(\cdot, \tilde{t})\right)^{2}+\left(\partial^{\alpha} v_{x x x}(\cdot, \tilde{t})\right)^{2}+\left(\partial^{\alpha} v_{y y}(\cdot, \tilde{t})\right)^{2}+\left(\partial^{\alpha} v_{t}(\cdot, \tilde{t})\right)^{2} \\
& \quad \leq C\|w\|_{Z_{t}^{|\alpha|} \mid\|v\|_{Z_{t}^{|\alpha|}}^{2}}
\end{aligned}
$$

We begin by taking $\alpha$ derivatives of (3.4). Our differentiated equation is

$$
\partial^{\alpha} v_{t}+\partial^{\alpha} v_{x x x}+\partial^{\alpha} v_{x}+\partial^{\alpha} \partial_{x}^{-1} v_{y y}+\partial^{\alpha}\left(w v_{x}\right)=0
$$

We then multiply (3.6) by $2\left(\partial^{\alpha} v\right)$ and integrate over $\mathbb{R}^{2}$. This implies

$$
\begin{aligned}
\partial_{t} \int\left(\partial^{\alpha} v(\cdot, \tilde{t})\right)^{2} & \leq C\left|\int \partial^{\alpha}\left(w v_{x}\right)\left(\partial^{\alpha} v\right)\right| \\
& \leq C\left|\int\left\{\left(\partial^{\alpha} w\right) v_{x}+\cdots+w\left(\partial^{\alpha} v_{x}\right)\right\}\left(\partial^{\alpha} v\right)\right|
\end{aligned}
$$

The remainder terms can be bounded as follows

$$
\begin{aligned}
\left|\int\left(\partial^{\alpha} w\right) v_{x}\left(\partial^{\alpha} v\right)\right| & \leq\left|v_{x}\right|_{L^{\infty}}\left(\int\left(\partial^{\alpha} w\right)^{2}\right)^{1 / 2}\left(\int\left(\partial^{\alpha} v\right)^{2}\right)^{1 / 2} \\
& \leq C\left(\int v_{x}^{2}+v_{x x x}^{2}+v_{x y}^{2}\right)^{1 / 2}\|w\|_{H^{|\alpha|} \mid}\|v\|_{H^{|\alpha|}} \\
& \leq C\|w\|_{Z_{t}^{|\alpha|}}\|v\|_{Z_{t}^{|\alpha|}}^{2}
\end{aligned}
$$


and

$$
\begin{aligned}
\left|\int w\left(\partial^{\alpha} v_{x}\right)\left(\partial^{\alpha} v\right)\right| & =C\left|\int w_{x}\left(\partial^{\alpha} v\right)^{2}\right| \\
& \leq C\left|w_{x}\right|_{L^{\infty}}\left(\int\left(\partial^{\alpha} v\right)^{2}\right) \\
& \leq C\left(\int w_{x}^{2}+w_{x x x}^{2}+w_{x y}^{2}\right)^{1 / 2}\|v\|_{Z_{t}^{|\alpha|}}^{2} \\
& \leq C\|w\|_{Z_{t}^{|\alpha|}}\|v\|_{Z_{t}^{|\alpha|} .}^{2}
\end{aligned}
$$

Therefore, we have

$$
\partial_{t} \int\left(\partial^{\alpha} v\right)^{2} \leq C\|w\|_{Z_{t}^{|\alpha|}}\|v\|_{Z_{t}^{|\alpha|}}^{2} .
$$

Next, we take three $x$ derivatives of (3.6), multiply by $2\left(\partial^{\alpha} v_{x x x}\right)$, and integrate over $\mathbb{R}^{2}$. Our inequality becomes

$$
\begin{aligned}
& \partial_{t} \int\left(\partial^{\alpha} v_{x x x}\right)^{2} \\
& \leq C\left|\int\left(\partial^{\alpha}\left(w v_{x}\right)_{x x x}\right)\left(\partial^{\alpha} v_{x x x}\right)\right| \\
& \leq C\left|\int \partial^{\alpha}\left(w_{x x x} v_{x}+2 w_{x x} v_{x x}+2 w_{x} v_{x x x}+w v_{x x x x}\right)\left(\partial^{\alpha} v_{x x x}\right)\right| \\
& \leq C\left|\int \partial^{\alpha}\left(w_{x x x} v_{x}\right)\left(\partial^{\alpha} v_{x x x}\right)\right|+C\left|\int \partial^{\alpha}\left(w_{x x} v_{x x}\right)\left(\partial^{\alpha} v_{x x x}\right)\right| \\
& \quad+C\left|\int_{\partial^{\alpha}}\left(w_{x} v_{x x x}\right)\left(\partial^{\alpha} v_{x x x}\right)\right|+C\left|\int \partial^{\alpha}\left(w v_{x x x x}\right)\left(\partial^{\alpha} v_{x x x}\right)\right| \\
& \leq A_{1}+A_{2}+A_{3}+A_{4} .
\end{aligned}
$$

We will look at terms $A_{1}-A_{4}$ below.

First, for $A_{1}$, we have

$$
\begin{aligned}
& \left|\int \partial^{\alpha}\left(w_{x x x} v_{x}\right)\left(\partial^{\alpha} v_{x x x}\right)\right| \\
& =\left|\int\left(\partial^{\alpha} w_{x x x}\right) v_{x}\left(\partial^{\alpha} v_{x x x}\right)+\cdots+w_{x x x}\left(\partial^{\alpha} v_{x}\right)\left(\partial^{\alpha} v_{x x x}\right)\right| \\
& \leq\left|v_{x}\right|_{L^{\infty}}\left(\int\left(\partial^{\alpha} w_{x x x}\right)^{2}\right)^{1 / 2}\left(\int\left(\partial^{\alpha} v_{x x x}\right)^{2}\right)^{1 / 2} \\
& +\cdots+\left|\partial^{\alpha} v_{x}\right|_{L^{\infty}}\left(\int w_{x x x}^{2}\right)^{1 / 2}\left(\int\left(\partial^{\alpha} v_{x x x}\right)^{2}\right)^{1 / 2} \\
& \leq\|v\|_{Z_{t}^{0}}\|w\|_{Z_{t}^{|\alpha|}}\|v\|_{Z_{t}^{|\alpha|}}+\cdots+\|v\|_{Z_{t}^{|\alpha|}}\|w\|_{Z_{t}^{0}}\|v\|_{Z_{t}^{|\alpha|}} \\
& \leq C\|w\|_{Z_{t}^{|\alpha|}}\|v\|_{Z_{t}^{|\alpha|}}^{2}
\end{aligned}
$$


For $A_{2}$,

$$
\left|\int \partial^{\alpha}\left(w_{x x} v_{x x}\right)\left(\partial^{\alpha} v_{x x x}\right)\right|=\left|\int\left\{\left(\partial^{\alpha} w_{x x}\right) v_{x x}+\cdots+w_{x x}\left(\partial^{\alpha} v_{x x}\right)\right\}\left(\partial^{\alpha} v_{x x x}\right)\right| .
$$

To bound these terms, we will use the following anisotropic imbedding found in [BIN]. For $2 \leq n<6$,

$$
\left(\int|u|^{n}\right)^{1 / n} \leq\left(\int u^{2}+u_{x}^{2}+\left(\partial_{x}^{-1} u_{y}\right)^{2}\right)^{1 / 2}
$$

We will look at the most difficult terms to bound below.

$$
\begin{aligned}
\left|\int\left(\partial^{\alpha} w_{x x}\right) v_{x x}\left(\partial^{\alpha} v_{x x x}\right)\right| \leq & \left(\int\left(\partial^{\alpha} w_{x x}\right)^{4}\right)^{1 / 4}\left(\int v_{x x}^{4}\right)^{1 / 4}\left(\int\left(\partial^{\alpha} v_{x x x}\right)^{2}\right)^{1 / 2} \\
\leq & \left(\int\left(\partial^{\alpha} w_{x x}\right)^{2}+\left(\partial^{\alpha} w_{x x x}\right)^{2}+\left(\partial^{\alpha} w_{x y}\right)^{2}\right)^{1 / 2} \\
& \cdot\left(\int v_{x x}^{2}+v_{x x x}^{2}+v_{x y}^{2}\right)^{1 / 2}\left(\int\left(\partial^{\alpha} v_{x x x}\right)^{2}\right)^{1 / 2} \\
\leq & \|w\|_{Z_{t}^{|\alpha|}}\|v\|_{Z_{t}^{|\alpha|}}^{2},
\end{aligned}
$$

while

$$
\begin{aligned}
\left|\int w_{x x}\left(\partial^{\alpha} v_{x x}\right)\left(\partial^{\alpha} v_{x x x}\right)\right| & =C\left|\int w_{x x x}\left(\partial^{\alpha} v_{x x}\right)^{2}\right| \\
& \leq C\left(\int w_{x x x}^{2}\right)^{1 / 2}\left(\int\left(\partial^{\alpha} v_{x x}\right)^{4}\right)^{1 / 2} \\
& \leq C\|w\|_{Z_{t}^{0}}\left(\int\left(\partial^{\alpha} v_{x x}\right)^{2}+\left(\partial^{\alpha} v_{x x x}^{2}\right)+\left(\partial^{\alpha} v_{x y}^{2}\right)\right)^{1 / 2} \\
& \leq C\|w\|_{Z_{t}^{|\alpha|}}\|v\|_{Z_{t}^{|\alpha|} .}^{2}
\end{aligned}
$$

Next, for $A_{3}$,

$$
\begin{aligned}
\left|\int \partial^{\alpha}\left(w_{x} v_{x x x}\right)\left(\partial^{\alpha} v_{x x x}\right)\right| & =\left|\int\left\{\left(\partial^{\alpha} w_{x}\right) v_{x x x}+\cdots+w_{x}\left(\partial^{\alpha} v_{x x x}\right)\right\}\left(\partial^{\alpha} v_{x x x}\right)\right| \\
& \leq\left|\partial^{\alpha} w_{x}\right|_{L^{\infty}}\left(\int v_{x x x}^{2}\right)^{1 / 2}\left(\int\left(\partial^{\alpha} v_{x x x}\right)^{2}\right)^{1 / 2} \\
& +\cdots+\left|w_{x}\right|_{L^{\infty}}\left(\int\left(\partial^{\alpha} v_{x x x}\right)^{2}\right) \\
& \leq C\|w\|_{Z_{t}^{|\alpha|}}\|v\|_{Z_{t}^{0}}\|v\|_{Z_{t}^{|\alpha|}}+\cdots+C\|w\|_{Z_{t}^{0}}\|v\|_{Z_{t}^{|\alpha|}}^{2} \\
& \leq C\|w\|_{Z_{t}^{|\alpha|} \mid}\|v\|_{Z_{t}^{|\alpha|} .}
\end{aligned}
$$


Lastly, we look at $A_{4}$,

$$
\left|\int \partial^{\alpha}\left(w v_{x x x x}\right)\left(\partial^{\alpha} v_{x x x}\right)\right|=\left|\int\left\{\left(\partial^{\alpha} w\right) v_{x x x x}+\cdots+w\left(\partial^{\alpha} v_{x x x x}\right)\right\}\left(\partial^{\alpha} v_{x x x}\right)\right| .
$$

The first term is handled below. If $\alpha=(0,0)$, then

$$
\begin{aligned}
\left|\int\left(\partial^{\alpha} w\right) v_{x x x x}\left(\partial^{\alpha} v_{x x x}\right)\right| & =\left|\int w v_{x x x x} v_{x x x}\right|=C\left|\int w_{x} v_{x x x}^{2}\right| \\
& \leq C\left|w_{x}\right|_{L^{\infty}}\left(\int v_{x x x}^{2}\right) \leq C\|w\|_{Z_{t}^{0}}\|v\|_{Z_{t}^{0}}^{2},
\end{aligned}
$$

while if $|\alpha|>0$, then

$$
\begin{aligned}
\left|\int\left(\partial^{\alpha} w\right) v_{x x x x}\left(\partial^{\alpha} v_{x x x}\right)\right| & \leq\left|\partial^{\alpha} w\right|_{L^{\infty}}\left(\int v_{x x x x}^{2}\right)^{1 / 2}\left(\int\left(\partial^{\alpha} v_{x x x}\right)^{2}\right)^{1 / 2} \\
& \leq\|w\|_{Z_{t}^{|\alpha|} \|}\|v\|_{Z_{t}^{|\alpha|}}^{2}
\end{aligned}
$$

The last term in $A_{4}$ is handled below,

$$
\begin{aligned}
\left|\int w\left(\partial^{\alpha} v_{x x x x}\right)\left(\partial^{\alpha} v_{x x x}\right)\right| & =C\left|\int w_{x}\left(\partial^{\alpha} v_{x x x}\right)^{2}\right| \leq C\left|w_{x}\right|_{L^{\infty}}\left(\int\left(\partial^{\alpha} v_{x x x}\right)^{2}\right) \\
& \leq C\|w\|_{Z_{t}^{0}}\|v\|_{Z_{t}^{|\alpha|}}^{2} \leq C\|w\|_{Z_{t}^{|\alpha|}}\|v\|_{Z_{t}^{|\alpha|}}^{2}
\end{aligned}
$$

Consequently, we conclude

$$
\partial_{t} \int\left(\partial^{\alpha} v_{x x x}\right)^{2} \leq C\|w\|_{Z_{t}^{|\alpha|}}\|v\|_{Z_{t}^{|\alpha|}}^{2}
$$

Next we take two $y$ derivatives of (3.6), multiply by $2\left(\partial^{\alpha} v_{y y}\right)$, and integrate over $\mathbb{R}^{2}$. Therefore, we have

$$
\begin{aligned}
\partial_{t} \int\left(\partial^{\alpha} v_{y y}\right)^{2} \leq & C\left|\int \partial^{\alpha}\left(w v_{x}\right)_{y y}\left(\partial^{\alpha} v_{y y}\right)\right| \\
\leq & C\left|\int \partial^{\alpha}\left(w_{y y} v_{x}+2 w_{y} v_{x y}+w v_{x y y}\right)\left(\partial^{\alpha} v_{y y}\right)\right| \\
\leq & C\left|\int \partial^{\alpha}\left(w_{y y} v_{x}\right)\left(\partial^{\alpha} v_{y y}\right)\right|+C\left|\int \partial^{\alpha}\left(w_{y} v_{x y}\right)\left(\partial^{\alpha} v_{y y}\right)\right| \\
& +C\left|\int \partial^{\alpha}\left(w v_{x y y}\right)\left(\partial^{\alpha} v_{y y}\right)\right| \\
\leq & B_{1}+B_{2}+B_{3} .
\end{aligned}
$$


First, we look at $B_{1}$,

$$
\begin{aligned}
\left|\int \partial^{\alpha}\left(w_{y y} v_{x}\right)\left(\partial^{\alpha} v_{y y}\right)\right|= & \left|\int\left\{\left(\partial^{\alpha} w_{y y}\right) v_{x}+\cdots+w_{y y}\left(\partial^{\alpha} v_{x}\right)\right\}\left(\partial^{\alpha} v_{y y}\right)\right| \\
\leq & C\left|v_{x}\right|_{L^{\infty}}\left(\int\left(\partial^{\alpha} w_{y y}\right)^{2}\right)^{1 / 2}\left(\int\left(\partial^{\alpha} v_{y y}\right)^{2}\right)^{1 / 2} \\
& +\cdots+C\left|\partial^{\alpha} v_{x}\right|_{L^{\infty}}\left(\int w_{y y}^{2}\right)^{1 / 2}\left(\int\left(\partial^{\alpha} v_{y y}\right)^{2}\right)^{1 / 2} \\
\leq & C\|v\|_{Z_{t}^{0}}\|w\|_{Z_{t}^{|\alpha|} \mid}\|v\|_{Z_{t}^{|\alpha|}}+\cdots+C\|v\|_{Z_{t}^{|\alpha|}}^{2}\|w\|_{Z_{t}^{0}} \\
\leq & C\|w\|_{Z_{t}^{|\alpha|} \mid}\|v\|_{Z_{t}^{|\alpha|} .}^{2}
\end{aligned}
$$

Next, for $B_{2}$,

$$
\begin{aligned}
\left|\int \partial^{\alpha}\left(w_{y} v_{x y}\right)\left(\partial^{\alpha} v_{y y}\right)\right| \\
=\left|\int\left\{\left(\partial^{\alpha} w_{y}\right) v_{x y}+\cdots+w_{y}\left(\partial^{\alpha} v_{x y}\right)\right\}\left(\partial^{\alpha} v_{y y}\right)\right| \\
\leq C\left|\partial^{\alpha} w_{y}\right|_{L^{\infty}}\left(\int v_{x y}^{2}\right)^{1 / 2}\left(\int\left(\partial^{\alpha} v_{y y}\right)^{2}\right)^{1 / 2} \\
\quad+\cdots+C\left|w_{y}\right|_{L^{\infty}}\left(\int\left(\partial^{\alpha} v_{x y}\right)^{2}\right)^{1 / 2}\left(\int\left(\partial^{\alpha} v_{y y}\right)^{2}\right)^{1 / 2} \\
\leq C\|w\|_{Z_{t}^{|\alpha|} \mid}\|v\|_{Z_{t}^{0}}\|v\|_{Z_{t}^{|\alpha|}}+\cdots+C\|w\|_{Z_{t}^{0}}\|v\|_{Z_{t}^{|\alpha|}}^{2} \\
\leq C\|w\|_{Z_{t}^{|\alpha|}}\|v\|_{Z_{t}^{|\alpha|}}^{2}
\end{aligned}
$$

Lastly, for $B_{3}$,

$$
\left|\int \partial^{\alpha}\left(w v_{x y y}\right)\left(\partial^{\alpha} v_{y y}\right)\right|=\left|\int\left\{\left(\partial^{\alpha} w\right) v_{x y y}+\cdots+w\left(\partial^{\alpha} v_{x y y}\right)\right\}\left(\partial^{\alpha} v_{y y}\right)\right| .
$$

We will look at the first and last of these terms below. The rest of these terms are handled similarly. For the first term, if $\alpha=(0,0)$, then we have

$$
\begin{aligned}
\left|\int\left(\partial^{\alpha} w\right) v_{x y y}\left(\partial^{\alpha} v_{y y}\right)\right| & =\left|\int w v_{x y y} v_{y y}\right|=C\left|\int w_{x} v_{y y}^{2}\right| \\
& \leq C\left|w_{x}\right|_{L^{\infty}}\left(\int v_{y y}^{2}\right) \leq C\|w\|_{Z_{t}^{0}}\|v\|_{Z_{t}^{0}}^{2},
\end{aligned}
$$

while for $|\alpha|>0$,

$$
\begin{aligned}
\left|\int\left(\partial^{\alpha} w\right) v_{x y y}\left(\partial^{\alpha} v_{y y}\right)\right| & \leq\left|\partial^{\alpha} w\right|_{L^{\infty}}\left(\int v_{x y y}^{2}\right)^{1 / 2}\left(\int\left(\partial^{\alpha} v_{y y}\right)^{2}\right)^{1 / 2} \\
& \leq\|w\|_{Z_{t}^{|\alpha|}}\|v\|_{Z_{t}^{|\alpha|}}^{2}
\end{aligned}
$$


The last term for $B_{3}$ is bounded as follows

$$
\begin{aligned}
\left|\int w\left(\partial^{\alpha} v_{x y y}\right)\left(\partial^{\alpha} v_{y y}\right)\right| & =C\left|\int w_{x}\left(\partial^{\alpha} v_{y y}\right)^{2}\right| \leq C\left|w_{x}\right|_{L^{\infty}}\left(\int\left(\partial^{\alpha} v_{y y}\right)^{2}\right) \\
& \leq C\|w\|_{Z_{t}^{|\alpha|} \mid} \mid v \|_{Z_{t}^{|\alpha|}}^{2} .
\end{aligned}
$$

Now apply one $t$ derivative to (3.6), multiply by $2\left(\partial^{\alpha} v_{t}\right)$, and integrate over $\mathbb{R}^{2}$. We arrive at the following inequality,

$$
\begin{aligned}
\partial_{t} \int\left(\partial^{\alpha} v_{t}\right)^{2} & \leq C\left|\int\left(\partial^{\alpha}\left(w v_{x}\right)_{t}\right)\left(\partial^{\alpha} v_{t}\right)\right| \\
& \leq C\left|\int \partial^{\alpha}\left(w_{t} v_{x}\right)\left(\partial^{\alpha} v_{t}\right)\right|+C\left|\int \partial^{\alpha}\left(w v_{x t}\right)\left(\partial^{\alpha} v_{t}\right)\right| \\
& \equiv D_{1}+D_{2} .
\end{aligned}
$$

First, for $D_{1}$ we have

$$
\begin{aligned}
& \left|\int \partial^{\alpha}\left(w_{t} v_{x}\right)\left(\partial^{\alpha} v_{t}\right)\right| \leq C\left|\int\left(\partial^{\alpha} w_{t}\right) v_{x}\left(\partial^{\alpha} v_{t}\right)\right| \\
& +\cdots+C\left|\int w_{t}\left(\partial^{\alpha} v_{x}\right)\left(\partial^{\alpha} v_{t}\right)\right| \\
& \leq C\left|v_{x}\right|_{L^{\infty}}\left(\int\left(\partial^{\alpha} w_{t}\right)^{2}\right)^{1 / 2}\left(\int\left(\partial^{\alpha} v_{t}\right)^{2}\right)^{1 / 2} \\
& +\left|\partial^{\alpha} v_{x}\right|_{L^{\infty}}\left(\int\left(w_{t}\right)^{2}\right)^{1 / 2}\left(\int\left(\partial^{\alpha} v_{t}\right)^{2}\right)^{1 / 2}
\end{aligned}
$$

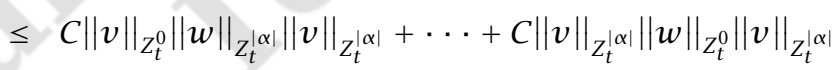

$$
\begin{aligned}
& \leq C\|w\|_{Z_{t}^{|\alpha|} \mid}\|v\|_{Z_{t}^{|\alpha|}}^{2} \text {. }
\end{aligned}
$$

Next we look at $D_{2}$. If $\alpha=(0,0)$, we have

$$
\begin{aligned}
& \left|\int w v_{x t} v_{t}\right| \\
& \quad=\left|\int w_{x} v_{t}^{2}\right| \leq\left|w_{x}\right|_{L^{\infty}}\left(\int v_{t}^{2}\right) \leq C\|w\|_{Z_{t}^{|\alpha|} \mid} \mid v \|_{Z_{t}^{|\alpha|}}^{2} .
\end{aligned}
$$

If $\alpha \neq(0,0)$, we have

$$
\begin{aligned}
\left|\int\left(\partial^{\alpha} w v_{x t}\right)\left(\partial^{\alpha} v_{t}\right)\right| & =C\left|\int\left(\partial^{\alpha} w\right) v_{x t}\left(\partial^{\alpha} v_{t}\right)\right|+\cdots+C\left|\int w\left(\partial^{\alpha} v_{x t}\right)\left(\partial^{\alpha} v_{t}\right)\right| \\
& \equiv D_{2}(a)+\cdots+D_{2}(b) .
\end{aligned}
$$

Now for $D_{2}(a)$, we use the following estimate,

$$
\begin{aligned}
\left|\int\left(\partial^{\alpha} w\right) v_{x t}\left(\partial^{\alpha} v_{t}\right)\right| & \leq C\left|\partial^{\alpha} w\right|_{L^{\infty}}\left(\int v_{x t}^{2}\right)^{1 / 2}\left(\int\left(\partial^{\alpha} v_{t}\right)^{2}\right)^{1 / 2} \\
& \leq C\|w\|_{Z_{t}^{|\alpha|} \mid}\|v\|_{Z_{t}^{\alpha \mid}}^{2} .
\end{aligned}
$$


While for $D_{2}(b)$, we use the following estimate,

$$
\begin{aligned}
\left|\int w\left(\partial^{\alpha} v_{x t}\right)\left(\partial^{\alpha} v_{t}\right)\right| & =C\left|\int w_{x}\left(\partial^{\alpha} v_{t}\right)^{2}\right| \leq C\left|w_{x}\right|_{L^{\infty}}\left(\int\left(\partial^{\alpha} v_{t}\right)^{2}\right) \\
& \leq C\|w\|_{Z_{t}^{|\alpha|} \mid}\|v\|_{Z_{t}^{|\alpha|} .}
\end{aligned}
$$

Therefore, for $0 \leq \tilde{t} \leq t$, we conclude that

$$
\begin{aligned}
& \partial_{t} \int\left(\partial^{\alpha} v(\cdot, \tilde{t})\right)^{2}+\left(\partial^{\alpha} v_{x x x}(\cdot, \tilde{t})\right)^{2}+\left(\partial^{\alpha} v_{y y}(\cdot, \tilde{t})\right)^{2}+\left(\partial^{\alpha} v_{t}(\cdot, \tilde{t})\right)^{2} \\
& \quad \leq C\|w\|_{Z_{t}^{|\alpha|}}\|v\|_{Z_{t}^{|\alpha|}}^{2} .
\end{aligned}
$$

Integrating with respect to $t$, we have

$$
\|v\|_{Z_{t}^{|\alpha|}}^{2} \leq\|v(\cdot, 0)\|_{H^{|\alpha|+3,|\alpha|+2}}^{2}+\left\|v_{t}(\cdot, 0)\right\|_{H^{|\alpha|}}^{2}+C t\|w\|_{Z_{t}^{|\alpha|}}\|v\|_{Z_{t}^{|\alpha|}}^{2},
$$

as desired.

Below we will prove the existence theorem for our linear equation (3.1).

Lemma 3.4. (Existence for linear equation) Given initial data

$$
\varphi \in \bigcap_{N \geq 0} H^{N}\left(\mathbb{R}^{2}\right), \quad \partial_{x}^{-1} \varphi_{y y} \in \bigcap_{N \geq 0} H^{N}\left(\mathbb{R}^{2}\right)
$$

there exists a unique solution of (3.1). The solution is defined in any time interval in which the coefficients are defined.

Proof. (Sketch) The linear equation which is to be solved at each iteration is of the form

$$
v_{t}+v_{x x x}+v_{x}+\partial_{x}^{-1} v_{y y}+b v_{x}=0
$$

where $b$ is a smooth, bounded coefficent. Fix a time $T>0$ and a constant $M>0$. Let

$$
\mathcal{L}=\partial_{t}+\partial_{x}^{3}+\partial_{x}+\partial_{x}^{-1} \partial_{y}^{2}+b \partial_{x}
$$


be defined on those functions $v \in X^{0}\left(\mathbb{R}^{2}\right)$. Recall that $v \in X^{0}\left(\mathbb{R}^{2}\right)$ means $v, v_{x x x},\left(\eta^{2} / \xi\right) \hat{v} \in L^{2}\left(\mathbb{R}^{2}\right)$. Introduce the bilinear form

$$
\langle g, h\rangle=\int_{0}^{T} \int e^{-M t} g h d x d y d t
$$

defined on those functions in $C\left([0, T] ; L^{2}\left(\mathbb{R}^{2}\right)\right)$ which vanish at $t=0$. By integrating by parts, we see that

$$
\begin{aligned}
\int \mathcal{L} v \cdot v d x d y & =\partial_{t} \int \frac{1}{2} v^{2} d x d y-\int \frac{1}{2} b_{x} v^{2} d x d y \\
& \geq \partial_{t} \int \frac{1}{2} v^{2} d x d y-c \int \frac{1}{2} v^{2} d x d y
\end{aligned}
$$

We then multiply by $e^{-M t}$ and integrate in time to obtain, for $v \in$ $C\left([0, T] ; X^{0}\left(\mathbb{R}^{2}\right)\right)$ with $v(x, y, 0) \equiv 0$,

$$
\langle\mathcal{L} v, v\rangle \geq e^{-M T} \int v^{2}(x, y, T) d x d y+(M-c) \int_{0}^{T} \int e^{-M t} v^{2} d x d y d t
$$

Thus, $\langle\mathcal{L} v, v\rangle \geq\langle v, v\rangle$ provided $M$ is chosen large enough.

Similarly, $\left\langle\mathcal{L}^{*} w, w\right\rangle \geq\langle w, w\rangle$ for all $w \in C\left([0, T] ; X^{0}\left(\mathbb{R}^{2}\right)\right)$ such that $w(x, y, T) \equiv 0$, where $\mathcal{L}^{*}$ denotes the formal adjoint of $\mathcal{L}$. Therefore, $\left\langle\mathcal{L}^{*} w, \mathcal{L}^{*} v\right\rangle$ is an inner product on $D \equiv\left\{w \in C\left([0, T] ; X^{0}\left(\mathbb{R}^{2}\right)\right) \mid w(x, y, T)\right.$ $\equiv 0\}$. Denote by $Y$ the completion of $D$ with respect to this inner product. By the Riesz representation theorem, there exists a unique solution $V \in Y$, such that for any $w \in D,\left\langle\mathcal{L}^{*} V, \mathcal{L}^{*} w\right\rangle=(\varphi, w(x, y, 0))$ where we used the fact that $(\varphi, w(x, y, 0))$ is a bounded linear functional on $D$. Then $v=\mathcal{L}^{*} V$ is a weak solution of $\mathcal{L} v=0, v_{0}=\varphi$ with $v \in L^{2}\left(\mathbb{R}^{2} \times[0, T]\right)$. To obtain higher regularity of the solution, we repeat the proof with higher derivatives included in the inner product. It is a standard approximation procedure to obtain a result for general initial data.

Now we want to improve our existence result. In particular, we want to show that the solution $u \in L^{\infty}\left([0, T] ; Y^{N}\left(\mathbb{R}^{2}\right)\right)$ found in Theorem 3.2 is in $L^{\infty}\left(\left[0, T^{\prime}\right] ; X^{N}\left(\mathbb{R}^{2}\right)\right)$ for a time $T^{\prime}$ depending only on $\|\varphi\|_{X^{0}\left(\mathbb{R}^{2}\right)}$.

Lemma 3.5. Let $u$ be the solution to our main equation in 
$L^{\infty}\left([0, T] ; Y^{N}\left(\mathbb{R}^{2}\right)\right)$. Then, for $0 \leq t \leq T$, we have

$$
\begin{aligned}
& \partial_{t}\left(\int u^{2}+\sum_{|\alpha| \leq N}\left\{\left(\partial^{\alpha} u_{x x x}\right)^{2}+\left(\partial^{\alpha}\left(\partial_{x}^{-1} u_{y y}\right)\right)^{2}\right\}\right) \\
& \quad \leq C\left(\int u^{2}+\sum_{|\alpha| \leq N}\left\{\left(\partial^{\alpha} u_{x x x}\right)^{2}+\left(\partial^{\alpha}\left(\partial_{x}^{-1} u_{y y}\right)\right)^{2}\right\}\right)^{3 / 2} .
\end{aligned}
$$

Proof. This inequality is derived as before using a priori estimates on smooth solutions $u$. Multiplying (2.1) by $u$ and integrating over $\mathbb{R}^{2}$, we see that the $L^{2}$ norm is conserved,

$$
\partial_{t} \int u^{2}=-2 \int u u_{x}=0
$$

Therefore, we only need to show that

$$
\int\left(\partial^{\alpha} u_{x x x}\right)^{2}+\left(\partial^{\alpha}\left(\partial_{x}^{-1} u_{y y}\right)\right)^{2} \leq\left(\int u^{2}+\sum_{|\alpha| \leq N}\left\{\left(\partial^{\alpha} u_{x x x}\right)^{2}+\left(\partial^{\alpha}\left(\partial_{x}^{-1} u_{y y}\right)\right)\right\}^{2}\right)^{3 / 2}
$$

For ease of notation, we consider the case $\alpha=(0,0)$. The case $\alpha \neq(0,0)$ is handled in the same way.

We begin by applying $\partial_{x}^{3}$ to $(2.1)$, multiplying by $2 u_{x x x}$, and integrating over $\mathbb{R}^{2}$. Upon doing so, we get

$\partial_{t} \int u_{x x x}^{2} \leq C\left|\int\left(u u_{x}\right)_{x x x} u_{x x x}\right|=C\left|\int\left\{3 u_{x x}^{2}+4 u_{x} u_{x x x}+u u_{x x x x}\right\} u_{x x x}\right|$

After integrating by parts, we see the first term on the right-hand side above vanishes and, thus, conclude

$$
\begin{aligned}
\partial_{t} \int u_{x x x}^{2} & \leq C\left|u_{x}\right|_{L^{\infty}} \int u_{x x x}^{2} \leq C\left(\int u_{x}^{2}+u_{x x x}^{2}+u_{x y}^{2}\right)^{1 / 2}\left(\int u_{x x x}^{2}\right) \\
& \leq C\left(\int u^{2}+u_{x x x}^{2}+\left(\partial_{x}^{-1} u_{y y}\right)^{2}\right)^{3 / 2} .
\end{aligned}
$$


We repeat the same procedure but now apply $\partial_{x}^{-1} \partial_{y}^{2}$ to (2.1) instead of $\partial_{x}^{3}$ and multiply by $2 \partial_{x}^{-1} u_{y y}$ instead of $2 u_{x x x}$. We get

$$
\begin{aligned}
& \partial_{t} \int\left(\partial_{x}^{-1} u_{y y}\right)^{2} \\
& \quad \leq C\left|\int\left(u^{2}\right)_{y y}\left(\partial_{x}^{-1} u_{y y}\right)\right|=C\left|\int\left\{u_{y y} u+u_{y}^{2}\right\}\left(\partial_{x}^{-1} u_{y y}\right)\right| \\
& \quad \leq C\left|\int u_{x}\left(\partial_{x}^{-1} u_{y y}\right)^{2}\right|+\left|\int u_{y}^{2}\left(\partial_{x}^{-1} u_{y y}\right)\right| \\
& \quad \leq C\left|u_{x}\right|_{L^{\infty}}\left(\int\left(\partial_{x}^{-1} u_{y y}\right)^{2}\right)+\left(\int u_{y}^{4}\right)^{1 / 2}\left(\int\left(\partial_{x}^{-1} u_{y y}\right)^{2}\right)^{1 / 2} \\
& \quad \leq C\left|u_{x}\right|_{L^{\infty}}\left(\int\left(\partial_{x}^{-1} u_{y y}\right)^{2}\right)+\left(\int u_{y}^{2}+u_{x y}^{2}+\left(\partial_{x}^{-1} u_{y y}\right)^{2}\right)\left(\int\left(\partial_{x}^{-1} u_{y y}\right)^{2}\right)^{1 / 2} \\
& \quad \leq C\left(\int u^{2}+u_{x x x}^{2}+\left(\partial_{x}^{-1} u_{y y}\right)^{2}\right)^{3 / 2},
\end{aligned}
$$

and, therefore, our lemma is proved.

Corollary 3.6. Let $\varphi \in Y^{N}\left(\mathbb{R}^{2}\right)$. Let $u$ be the solution with initial data $\varphi$. Denote by $0<T<\infty$ the life span of this solution in $Y^{N}\left(\mathbb{R}^{2}\right)$. Then there exists $0<T^{\prime} \leq T$, depending only on the norm of $\varphi \in X^{N}\left(\mathbb{R}^{2}\right)$ such that $u \in L^{\infty}\left(\left[0, T^{\prime}\right] ; X^{N}\left(\mathbb{R}^{2}\right)\right)$.

Proof. From Lemma 3.5 we have the differential inequality,

$$
\begin{aligned}
& \partial_{t}\left(\int u^{2}+\sum_{|\alpha| \leq N}\left(\partial^{\alpha} u_{x x x}\right)^{2}+\left(\partial^{\alpha}\left(\partial_{x}^{-1} u_{y y}\right)\right)^{2}\right) \\
& \quad \leq C\left(\int u^{2}+\sum_{|\alpha| \leq N}\left(\partial^{\alpha} u_{x x x}\right)^{2}+\left(\partial^{\alpha}\left(\partial_{x}^{-1} u_{y y}\right)\right)^{2}\right)^{3 / 2} .
\end{aligned}
$$

Let

$$
q(t)=\int\left(u^{2}+\sum_{|\alpha| \leq N}\left(\partial^{\alpha} u_{x x x}\right)^{2}+\left(\partial^{\alpha}\left(\partial_{x}^{-1} u_{y y}\right)\right)^{2}\right) \equiv\|u\|_{X^{N}}^{2} .
$$

Then the differential inequality may be stated $q^{\prime}(t) \leq(q(t))^{3 / 2}$. Integrating this inequality, we see that $q(t)^{1 / 2} \leq C /\left(q(0)^{-1 / 2}-t\right)$ and, therefore, we get a lower bound on the time of existence of $q(t)$ depending only on $q(0)$. 
Corollary 3.7. Let $\varphi \in X^{N}\left(\mathbb{R}^{2}\right)$ for some $N \geq 0$ and let $\varphi^{(n)}$ be a sequence converging to $\varphi$ in $X^{N}\left(\mathbb{R}^{2}\right)$. Let $u$ and $u^{(n)}$ be the corresponding unique solutions, given by Theorems 3.1 and 3.2 and Corollary 3.6, in $L^{\infty}\left([0, T] ; X^{N}\left(\mathbb{R}^{2}\right)\right)$ for a time $T$ depending only on $\sup _{n}\left\|\varphi^{(n)}\right\|_{X^{0}}$. Then $u^{(n)}-u$ weak* in $L^{\infty}\left([0, T] ; X^{N}\left(\mathbb{R}^{2}\right)\right)$.

Proof. By assumption, $u^{(n)} \in L^{\infty}\left([0, T] ; X^{N}\left(\mathbb{R}^{2}\right)\right)$, and, therefore, there exists a weak* convergent subsequence, still denoted $\left\{u^{(n)}\right\}$ such that $u^{(n)}$ $v$ weak ${ }^{*}$ in $L^{\infty}\left([0, T] ; X^{N}\left(\mathbb{R}^{2}\right)\right) \subset L^{\infty}\left([0, T] ; H^{1}\left(\mathbb{R}^{2}\right)\right)$. In addition, by equation (2.1), $u^{(n)} \in L^{\infty}\left([0, T] ; X^{N}\left(\mathbb{R}^{2}\right)\right)$ implies $u_{t}^{(n)} \in L^{\infty}\left([0, T] ; L^{2}\left(\mathbb{R}^{2}\right)\right)$. Therefore, by Aubin's compactness theorem, $u^{(n)} \rightarrow v$ strongly in $L^{2}\left([0, T] ; H_{\text {loc }}^{1 / 2}\left(\mathbb{R}^{2}\right)\right)$. Now we just need to show that each term in (2.1) converges to its correct limit, and, thus $u_{t}^{(n)} \rightarrow v_{t}$ for some solution $v \in$ $L^{\infty}\left([0, T] ; X^{N}\left(\mathbb{R}^{2}\right)\right)$. Then, by Theorem 3.1 on uniqueness, we conclude that $v \equiv u$.

The only thing we need to show is that the nonlinear term converges to its correct limit, namely, that $u^{(n)} u_{x}^{(n)} \rightarrow v v_{x}$. We know that

$$
\begin{array}{lll}
u_{x}^{(n)} \rightarrow v & \text { weak } * \text { in } & L^{\infty}\left([0, T] ; H^{1}\left(\mathbb{R}^{2}\right)\right), \text { and } \\
u^{(n)} \rightarrow v & \text { strongly in } & L^{2}\left([0, T] ; H_{\mathrm{loc}}^{1 / 2}\left(\mathbb{R}^{2}\right)\right) .
\end{array}
$$

Therefore, their product converges in $L^{2}\left([0, T] ; L_{\mathrm{loc}}^{1}\left(\mathbb{R}^{2}\right)\right)$. Clearly, the linear terms also converge in $L^{2}\left([0, T] ; L_{\text {loc }}^{1}\left(\mathbb{R}^{2}\right)\right)$, and, therefore, we conclude that $u_{t}^{(n)} \rightarrow v_{t}$ in $L^{2}\left([0, T] ; L_{\text {loc }}^{1}\left(\mathbb{R}^{2}\right)\right)$ for some solution $v$. We also know that $v \in L^{\infty}\left([0, T] ; X^{N}\left(\mathbb{R}^{2}\right)\right)$. Therefore, by Theorem 3.1, $v \equiv u$.

4. Weighted Estimates. In the previous section, we proved the existence of a solution $u$ to $(2.1)$ in $L^{\infty}\left([0, T] ; X^{N}\left(\mathbb{R}^{2}\right)\right)$ for given initial data $\varphi$ in $X^{N}\left(\mathbb{R}^{2}\right)$. In this section, we prove that if, in addition, our initial data $\varphi$ lies in some weighted space, $\tilde{H}^{K}\left(W_{0, i, 0}\right)$, for some integers $K \geq 0, i \geq 2$, then our solution $u$ also lies in $L^{\infty}\left([0, T] ; \tilde{H}^{K}\left(W_{0, i, 0}\right)\right)$. This property is known as a "persistence" property of the initial data. While of interest in its own right, it also provides a basis for starting the induction for Theorem 2.1, which shows the gain in regularity. 
Theorem 4.1. (Existence in a weighted space) Assume $u$ is the solution to (2.1) in $L^{\infty}\left([0, T] ; X^{0}\left(\mathbb{R}^{2}\right)\right)$ with initial data $\varphi(x, y) \in X^{0}\left(\mathbb{R}^{2}\right)$ such that $\varphi$ also lies in the weighted space $\widetilde{H}^{K}\left(W_{0, i, 0}\right)$ for some integers $K \geq 0, i \geq 2$. Then

$$
u \in L^{\infty}\left([0, T] ; X^{0}\left(\mathbb{R}^{2}\right) \cap \widetilde{H}^{K}\left(W_{0, i, 0}\right),\right.
$$

and

$$
\int_{0}^{T} \int \eta\left(\partial^{\gamma} u_{x}\right)^{2}+\int_{0}^{T} \int \eta\left(\partial^{\gamma}\left(\partial_{x}^{-1} u_{y}\right)\right)^{2} \leq C,
$$

for $\gamma=\left(\gamma_{1}, \gamma_{2}\right) \in \mathbb{Z}^{+} \times \mathbb{Z}^{+}, \gamma_{1}+2 \gamma_{2} \leq K$, where $\eta$ is a weight function in $W_{\sigma, i-1,0}$ for $\sigma>0$ arbitrary, and $C$ depends only on $T$ and the norm of $\varphi \in X^{0}\left(\mathbb{R}^{2}\right) \cap \tilde{H}^{K}\left(W_{0, i, 0}\right)$.

Proof. We will prove this result by induction on $\beta$, for $0 \leq \beta \leq K$. As before, we need to derive $a$ priori estimates for smooth solutions $u$, which depend only on the norms of $u \in L^{\infty}\left([0, T] ; X^{0}\left(\mathbb{R}^{2}\right)\right)$ and $\varphi \in \tilde{H}^{K}\left(W_{0, i, 0}\right)$. Then, we can apply convergence arguments to show that the result holds true for general solutions. In order to do so, we need to approximate general solutions $u \in X^{0}\left(\mathbb{R}^{2}\right)$ by smooth solutions and approximate general weight functions $\xi$ in $W_{0, i, 0}$ by smooth, bounded weight functions. We have discussed approximating solutions in the previous section, so we will concentrate on the approximation of the weight functions here.

We begin by taking a sequence of bounded weight functions $\eta_{\delta}$, which decay as $|x| \rightarrow \infty$ and which approximate $\eta \in W_{\sigma, i-1,0}$ from below, uniformly on any half-line $(-\infty, c)$. Let

$$
\xi_{\delta}(x, t) \equiv 1+\int_{-\infty}^{x} \eta_{\delta}(z, t) d z .
$$

Therefore, the functions $\xi_{\delta}$ are bounded weight functions which approximate a weight function $\xi \in W_{0, i, 0}$ from below, uniformly on compact sets.

As we did in the proof of Lemma 2.2, for the $\beta^{\text {th }}$ induction step, we take $\alpha$ derivatives of (2.1), where $\alpha_{1}+2 \alpha_{2}=\beta$, multiply our differentiated equation by $2 \xi_{\delta}\left(\partial^{\alpha} u\right)$, and integrate over $\mathbb{R}^{2}$. Using the fact that $\left(\xi_{\delta}\right)_{t},\left(\xi_{\delta}\right)_{x} \leq C \xi_{\delta}$, we get the following main identity,

$$
\begin{gathered}
\partial_{t} \int \xi_{\delta}\left(\partial^{\alpha} u\right)^{2}+3 \int\left(\xi_{\delta}\right)_{x}\left(\partial^{\alpha} u_{x}\right)^{2}+\int\left(\xi_{\delta}\right)_{x}\left(\partial^{\alpha}\left(\partial_{x}^{-1} u_{y}\right)\right)^{2} \\
\quad \leq \int \xi_{\delta}\left(\partial^{\alpha} u\right)^{2}+\left|\int 2 \xi_{\delta}\left(\partial^{\alpha} u\right) \partial^{\alpha}\left(u u_{x}\right)\right| .
\end{gathered}
$$


On each step of the induction, we must now prove $a$ priori bounds on the remainder terms, depending only on the norm of $\varphi \in X^{0}\left(\mathbb{R}^{2}\right) \cap \tilde{H}^{\beta}\left(W_{0, i, 0}\right)$. The first few steps require some care, so we do these separately. The case $\beta \geq 4$ follows from Lemma 4.2 below.

$\triangleright$ The case $\beta=0$. In particular, we note that for the case $\beta=0$, the third term on the left-hand side of (4.3) can be justified by the use of the Fourier transform and an approximation argument. For the first step of the induction, $\beta=0$, we have,

$$
\begin{aligned}
\left|\int \xi_{\delta}\left(\partial^{\alpha} u\right) \partial^{\alpha}\left(u u_{x}\right)\right| & =C\left|\int \xi_{\delta} u^{2} u_{x}\right| \leq C\left|u_{x}\right|_{L^{\infty}}\left(\int \xi_{\delta} u^{2}\right) \\
& \leq C\|u\|_{X^{0}}\left(\int \xi_{\delta} u^{2}\right) \leq C\left(\int \xi_{\delta} u^{2}\right) .
\end{aligned}
$$

Combining this estimate with (4.3) for the case $\alpha=(0,0)$, we have, for $0 \leq t \leq T$,

$$
\int \xi_{\delta}(\cdot, t) u^{2} \leq \int \xi_{\delta}(\cdot, 0) \varphi^{2}+C \int_{0}^{t} \int \xi_{\delta} u^{2} \leq C+C \int_{0}^{t} \int \xi_{\delta} u^{2} .
$$

Therefore, by Gronwall's inequality,

$$
\sup _{0 \leq t \leq T} \int \xi_{\delta} u^{2}+3 \int_{0}^{T} \int\left(\xi_{\delta}\right)_{x} u_{x}^{2}+\int_{0}^{T} \int\left(\xi_{\delta}\right)_{x}\left(\partial_{x}^{-1} u_{y}\right)^{2} \leq C,
$$

where $C$ does not depend on $\delta$, but only on $T$ and the norm of $\varphi \in X^{0}\left(\mathbb{R}^{2}\right) \cap$ $\widetilde{H}^{0}\left(W_{0, i, 0}\right)$. Consequently, we can pass to the limit and conclude,

$$
\sup _{0 \leq t \leq T} \int \xi u^{2}+3 \int_{0}^{T} \int \eta u_{x}^{2}+\int_{0}^{T} \int \eta\left(\partial_{x}^{-1} u_{y}\right)^{2} \leq C .
$$

$\triangleright$ The case $\beta=1$. For $\beta=1$, we have

$$
\begin{aligned}
\left|\int \xi_{\delta}\left(\partial^{\alpha} u\right) \partial^{\alpha}\left(u u_{x}\right)\right| & =\left|\int \xi_{\delta}\left(u_{x}^{3}+u u_{x} u_{x x}\right)\right| \\
& \leq C\left|u_{x}\right|_{L^{\infty}}\left(\int \xi_{\delta} u_{x}^{2}\right)+C\left|\int\left[\xi_{\delta} u\right]_{x} u_{x}^{2}\right| \\
& \leq C\left(|u|_{L^{\infty}}+\left|u_{x}\right|_{L^{\infty}}\right)\left(\int \xi_{\delta} u_{x}^{2}\right) \\
& \leq C\|u\|_{X^{0}}\left(\int \xi_{\delta} u_{x}^{2}\right) .
\end{aligned}
$$


We combine this estimate with (4.3) for the case $\alpha=(1,0)$ and use Gronwall's inequality, to conclude

$$
\sup _{0 \leq t \leq T} \int \xi_{\delta} u_{x}^{2}+\int_{0}^{T} \int\left(\xi_{\delta}\right)_{x} u_{x x}^{2}+\int_{0}^{T} \int\left(\xi_{\delta}\right)_{x} u_{y}^{2} \leq C,
$$

where $C$ does not depend on $\delta$, but only on $T$ and the norm of $\varphi \in X^{0}\left(\mathbb{R}^{2}\right) \cap$ $\tilde{H}^{1}\left(W_{0, i, 0}\right)$. Taking the limit as $\delta \rightarrow \infty$, we get the desired estimate,

$$
\sup _{0 \leq t \leq T} \int \xi u_{x}^{2}+\int_{0}^{T} \int \eta u_{x x}^{2}+\int_{0}^{T} \int \eta u_{y}^{2} \leq C .
$$

$\triangleright$ The case $\beta=2$. For $\beta=2$, we have two cases: $\alpha=(2,0)$ or $\alpha=$ $(0,1)$.

$\triangleright$ The subcase $\alpha=(2,0)$. First, for the case $\alpha=(2,0)$, our remainder terms are

$$
\begin{aligned}
\left|\int \xi_{\delta}\left(\partial^{\alpha} u\right) \partial^{\alpha}\left(u u_{x}\right)\right| & =\left|\int \xi_{\delta} u_{x x}\left\{3 u_{x} u_{x x}+u u_{x x x}\right\}\right| \\
& \leq C\left|u_{x}\right|_{L^{\infty}}\left(\int \xi_{\delta} u_{x x}^{2}\right)+C\left|\int\left[\xi_{\delta} u\right]_{x} u_{x x}^{2}\right| \\
& \leq C\left(|u|_{L^{\infty}}+\left|u_{x}\right|_{L^{\infty}}\right)\left(\int \xi_{\delta} u_{x x}^{2}\right) \\
& \leq C\|u\|_{X^{0}}\left(\int \xi_{\delta} u_{x x}^{2}\right) .
\end{aligned}
$$

As before, we use the estimates on our remainder terms along with our main identity (4.3) and the fact that the bounds do not depend on $\delta$ to conclude that

$$
\sup _{0 \leq t \leq T} \int \xi u_{x x}^{2}+\int_{0}^{T} \int \eta u_{x x x}^{2}+\int_{0}^{T} \int \eta u_{x y}^{2} \leq C,
$$

where $C$ depends only on the norm of $\varphi \in X^{0}\left(\mathbb{R}^{2}\right) \cap \tilde{H}^{2}\left(W_{0, i, 0}\right)$.

$\triangleright$ The subcase $\alpha=(0,1)$. Similarly, for the case $\alpha=(0,1)$, our remainder terms are

$$
\begin{aligned}
\left|\int \xi_{\delta}\left(\partial^{\alpha} u\right) \partial^{\alpha}\left(u u_{x}\right)\right| & =\left|\int \xi_{\delta} u_{y}\left\{u_{y} u_{x}+u u_{x y}\right\}\right| \\
& \leq C\left(|u|_{L^{\infty}}+\left|u_{x}\right|_{L^{\infty}}\right)\left(\int \xi_{\delta} u_{y}^{2}\right)
\end{aligned}
$$


so we can conclude,

$$
\sup _{0 \leq t \leq T} \int \xi u_{y}^{2}+\int_{0}^{T} \int \eta u_{x y}^{2}+\int_{0}^{T} \int \eta\left(\partial_{x}^{-1} u_{y y}\right)^{2} \leq C
$$

where $C$ depends only on $T$ and the norm of $\varphi \in X^{0}\left(\mathbb{R}^{2}\right) \cap \tilde{H}^{2}\left(W_{0, i, 0}\right)$.

$\triangleright$ The case $\beta=3$. For $\beta=3$, we have two cases: $\alpha=(3,0)$ and $\alpha=(1,1)$. For these estimates, we again use (3.7), which states that, for $2 \leq n<6$,

$$
\left(\int|u|^{n}\right)^{1 / n} \leq\left(\int u^{2}+u_{x}^{2}+\left(\partial_{x}^{-1} u_{y}\right)^{2}\right)^{1 / 2}
$$

$\triangleright$ The subcase $\alpha=(3,0)$. For $\alpha=(3,0)$ our remainder terms are

$$
\left|\int \xi_{\delta}\left(\partial^{\alpha} u\right) \partial^{\alpha}\left(u u_{x}\right)\right|=\left|\int \xi_{\delta} u_{x x x}\left\{3 u_{x x}^{2}+4 u_{x} u_{x x x}+u u_{x x x x}\right\}\right| .
$$

We will look at each of these terms below. First, for $\int \xi_{\delta} u_{x x}^{2} u_{x x x}$, we will consider $x>1$ and $x<-1$ separately. We will again use the notation

$$
A=\{x>1\} \times \mathbb{R}, \quad B=\{x<-1\} \times \mathbb{R} .
$$

For the case $x>1$,

$$
\begin{aligned}
& \left|\int_{A} \xi_{\delta} u_{x x}^{2} u_{x x x}\right| \\
& \quad \leq\left(\int_{A} \xi_{\delta} u_{x x}^{4}\right)^{1 / 2}\left(\int_{A} \xi_{\delta} u_{x x x}^{2}\right)^{1 / 2} \leq\left(\int_{A}\left(\xi_{\delta}^{1 / 4} u_{x x}\right)^{4}\right)^{1 / 2}\left(\int_{A} \xi_{\delta} u_{x x x}^{2}\right)^{1 / 2} \\
& \quad \leq\left(\int_{A}\left(\left[\xi_{\delta}^{1 / 4} u_{x}\right]_{x}\right)^{4}\right)^{1 / 2}\left(\int_{A} \xi_{\delta} u_{x x x}^{2}\right)^{1 / 2} \\
& \quad \leq\left(\int_{A}\left(\left[\xi_{\delta}^{1 / 4} u_{x}\right]_{x}\right)^{2}+\left(\left[\xi_{\delta}^{1 / 4} u_{x}\right]_{x x}\right)^{2}+\left(\left[\xi_{\delta}^{1 / 4} u_{x}\right]_{y}\right)^{2}\right)\left(\int_{A} \xi_{\delta} u_{x x x}^{2}\right)^{1 / 2} \\
& \quad \leq\left(\int_{A} \xi_{\delta}^{1 / 2}\left\{u_{x}^{2}+u_{x x x}^{2}+u_{x y}^{2}\right\}\right)\left(\int_{A} \xi_{\delta} u_{x x x}^{2}\right)^{1 / 2} \\
& \quad \leq C\left(\int_{A}\left(\xi_{\delta}\right)_{x}\left\{u_{x}^{2}+u_{x x x}^{2}+u_{x y}^{2}\right\}\right)\left(1+\int_{A} \xi_{\delta} u_{x x x}^{2}\right),
\end{aligned}
$$


using the fact that for $x>1, \xi_{\delta}^{1 / 2} \leq\left(\xi_{\delta}\right)_{x}$, for $i \geq 2$. Next, for $x<-1$, we use the fact that $\xi_{\delta} \approx C$ to show

$$
\begin{aligned}
\left|\int_{B} \xi_{\delta} u_{x x}^{2} u_{x x x}\right| & \leq C\left|\int_{B} u_{x x}^{2} u_{x x x}\right| \leq C\left(\int_{B} u_{x x}^{4}\right)^{1 / 2}\left(\int_{B} u_{x x x}^{2}\right)^{1 / 2} \\
& \leq C\left(\int_{B} u_{x x}^{2}+u_{x x x}^{2}+u_{x y}^{2}\right)\left(\int_{B} u_{x x x}^{2}\right)^{1 / 2} \\
& \leq C\|u\|_{X^{0}}\left(\int_{B} u_{x x x}^{2}\right) \leq C\|u\|_{X^{0}}\left(\int_{B} \xi_{\delta} u_{x x x}^{2}\right) .
\end{aligned}
$$

For our second term,

$$
\left|\int \xi_{\delta} u_{x} u_{x x x}^{2}\right| \leq C\left|u_{x}\right|_{L^{\infty}}\left(\int \xi_{\delta} u_{x x x}^{2}\right) \leq C\|u\|_{X^{0}}\left(\int \xi_{\delta} u_{x x x}^{2}\right) .
$$

Lastly,

$$
\begin{aligned}
\left|\int \xi_{\delta} u u_{x x x} u_{x x x x}\right| & =C\left|\int\left[\xi_{\delta} u\right]_{x} u_{x x x}^{2}\right| \leq C\left(|u|_{L^{\infty}}+\left|u_{x}\right|_{L^{\infty}}\right)\left(\int \xi_{\delta} u_{x x x}^{2}\right) \\
& \leq C\|u\|_{X^{0}}\left(\int \xi_{\delta} u_{x x x}^{2}\right) .
\end{aligned}
$$

Combining these estimates with (4.3) for the case $\alpha=(3,0)$, yields

$$
\begin{aligned}
\int \xi_{\delta}(\cdot, t) u_{x x x}^{2} & \leq \int \xi_{\delta}(\cdot, 0) \varphi_{x x x}^{2}+C \\
& +C \int_{0}^{t}\left\{\left(1+\int\left(\xi_{\delta}\right)_{x}\left\{u_{x}^{2}+u_{x x x}^{2}+u_{x y}^{2}\right\}\right)\left(\int \xi_{\delta} u_{x x x}^{2}\right)\right\} d t
\end{aligned}
$$

for $0 \leq t \leq T$. Now we will look at the case $\alpha=(1,1)$ and combine the estimates for $\alpha=(3,0)$ and $\alpha=(1,1)$ to show that for $\varphi$ in $X^{0}\left(\mathbb{R}^{2}\right) \cap$ $\tilde{H}^{3}\left(W_{0, i, 0}\right), u$ is in $L^{\infty}\left([0, T] ; \tilde{H}^{3}\left(W_{0, i, 0}\right)\right)$.

$\triangleright$ The case $\alpha=(1,1)$. For the case $\alpha=(1,1)$, the remainder terms are

$$
\left|\int \xi_{\delta}\left(\partial^{\alpha} u\right) \partial^{\alpha}\left(u u_{x}\right)\right|=\left|\int \xi_{\delta} u_{x y}\left\{2 u_{x} u_{x y}+u_{y} u_{x x}+u u_{x x y}\right\}\right| .
$$

We can bound these terms as follows. First,

$$
\left|\int \xi_{\delta} u_{x} u_{x y}^{2}\right| \leq C\left|u_{x}\right|_{L^{\infty}}\left(\int \xi_{\delta} u_{x y}^{2}\right) \leq C\|u\|_{X^{0}}\left(\int \xi_{\delta} u_{x y}^{2}\right) .
$$


For the next term, $\int \xi_{\delta} u_{y} u_{x x} u_{x y}$, we will break it up into the cases when $x>1$ and when $x<-1$. For $x>1$, we have:

$$
\begin{aligned}
& \left|\int_{A} \xi_{\delta} u_{y} u_{x x} u_{x y}\right| \\
& \leq\left(\int_{A} \xi_{\delta}^{1 / 2} u_{y}^{2}\right)^{1 / 2}\left(\int_{A} \xi_{\delta}^{2} u_{x x}^{4}\right)^{1 / 4}\left(\int_{A} \xi_{\delta} u_{x y}^{4}\right)^{1 / 4} \\
& \leq\left(\int_{A} \xi_{\delta}^{1 / 2} u_{y}^{2}\right)^{1 / 2}\left(\int_{A} \xi_{\delta}\left\{u_{x}^{2}+u_{x x x}^{2}+u_{x y}^{2}\right\}\right)^{1 / 2} \\
& \quad \times\left(\int_{A} \xi_{\delta}^{1 / 2}\left\{u_{y}^{2}+u_{x x y}^{2}+u_{y y}^{2}\right\}\right)^{1 / 2} \\
& \leq \frac{C}{\varepsilon}\left(\int_{A}\left(\xi_{\delta}\right)_{x} u_{y}^{2}\right)\left(\int_{A} \xi_{\delta}\left\{u_{x x x}^{2}+u_{x y}^{2}\right\}\right)+\varepsilon\left(\int_{A}\left(\xi_{\delta}\right)_{x}\left\{u_{y}^{2}+u_{x x y}^{2}+u_{y y}^{2}\right\}\right),
\end{aligned}
$$

where we have used the fact that $\left(\xi_{\delta}\right)^{1 / 2} \leq C\left(\xi_{\delta}\right)_{x}$ for $i \geq 2$. For $x<-1$, we have

$$
\begin{aligned}
& \left|\int_{B} \xi_{\delta} u_{y} u_{x x} u_{x y}\right| \\
\leq & C\left|\int_{B} u_{y} u_{x x} u_{x y}\right| \leq C\left(\int_{B} u_{y}^{4}\right)^{1 / 4}\left(\int_{B} u_{x x}^{4}\right)^{1 / 4}\left(\int_{B} u_{x y}^{2}\right)^{1 / 2} \\
\leq & C\left(\int_{B} u_{y}^{2}+u_{x y}^{2}+\left(\partial_{x}^{-1} u_{y y}\right)^{2}\right)^{1 / 2}\left(\int_{B} u_{x x}^{2}+u_{x x x}^{2}+u_{x y}^{2}\right)^{1 / 2}\left(\int_{B} u_{x y}^{2}\right)^{1 / 2} \\
\leq & C\|u\|_{X^{0}}^{3} .
\end{aligned}
$$

Lastly,

$$
\begin{aligned}
\left|\int \xi_{\delta} u u_{x x y} u_{x y}\right| & =C\left|\int\left[\xi_{\delta} u\right]_{x} u_{x y}^{2}\right| \leq C\left(|u|_{L^{\infty}}+\left|u_{x}\right|_{L^{\infty}}\right)\left(\int \xi_{\delta} u_{x y}^{2}\right) \\
& \leq C\|u\|_{X^{0}}\left(\int \xi_{\delta} u_{x y}^{2}\right) \leq C\left(\int \xi_{\delta} u_{x y}^{2}\right) .
\end{aligned}
$$

Combining these estimates with (4.3) for the case $\alpha=(1,1)$ and bringing the $\varepsilon$ terms to the left-hand side, we conclude

$$
\begin{aligned}
\int \xi_{\delta}(\cdot, t) u_{x y}^{2} \leq & \int \xi_{\delta}(\cdot, 0) \varphi_{x y}^{2}+C \\
& +C \int_{0}^{t}\left\{\left(1+\int\left(\xi_{\delta}\right)_{x} u_{y}^{2}\right)\left(\int \xi_{\delta}\left\{u_{x x x}^{2}+u_{x y}^{2}\right\}\right)\right\} d t
\end{aligned}
$$

for $0 \leq t \leq T$. 
Now combining estimate $(4.4)$ (the case $\alpha=(3,0)$ ) with (4.5) (the case $\alpha=(1,1))$, we have

$$
\begin{aligned}
& \int \xi_{\delta}(\cdot, t)\left\{u_{x x x}^{2}+u_{x y}^{2}\right\} \\
& \leq \int \xi_{\delta}(\cdot, 0)\left\{\varphi_{x x x}^{2}+\varphi_{x y}^{2}\right\} \\
& \quad+C \int_{0}^{t}\left\{\left(1+\int\left(\xi_{\delta}\right)_{x}\left\{u_{x x x}^{2}+u_{x y}^{2}+u_{x}^{2}+u_{y}^{2}\right\}\right)\left(\int \xi_{\delta}\left\{u_{x x x}^{2}+u_{x y}^{2}\right\}\right)\right\} d t .
\end{aligned}
$$

Gronwall's inequality implies

$$
\text { (4.6) } \int \xi_{\delta}\left\{u_{x x x}^{2}+u_{x y}^{2}\right\} \leq C \exp \left(\int_{0}^{t}\left\{1+\int\left(\xi_{\delta}\right)_{x}\left\{u_{x x x}^{2}+u_{x y}^{2}+u_{x}^{2}+u_{y}^{2}\right\}\right\} d t\right) \text {. }
$$

However, we know that the terms on the right-hand side of (4.6) were all bounded on the previous step of the induction. Therefore, we can conclude that

$$
\begin{gathered}
\sup _{0 \leq t \leq T} \int \xi_{\delta}\left\{u_{x x x}^{2}+u_{x y}^{2}\right\}+\int_{0}^{T} \int\left(\xi_{\delta}\right)_{x} u_{x x x x}^{2} \\
+\int_{0}^{T} \int\left(\xi_{\delta}\right)_{x} u_{x x y}^{2}+\int_{0}^{T} \int\left(\xi_{\delta}\right)_{x} u_{y y}^{2} \leq C,
\end{gathered}
$$

where $C$ depends only on $T$ and the norm of $\varphi \in X^{0}\left(\mathbb{R}^{2}\right) \cap \tilde{H}^{3}\left(W_{0, i, 0}\right)$. Thus, we can take the limit as $\delta \rightarrow 0$ and get the desired estimate,

$$
\sup _{0 \leq t \leq T} \int \xi\left\{u_{x x x}^{2}+u_{x y}^{2}\right\}+\int_{0}^{T} \int \eta u_{x x x x}^{2}+\int_{0}^{T} \int \eta u_{x x y}^{2}+\int_{0}^{T} \int \eta u_{y y}^{2} \leq C .
$$

$\triangleright$ The case $\beta \geq 4$. For $K \geq \beta \geq 4,0 \leq t \leq T$, we use Lemma 4.2 below, where we prove that

$$
\sum_{\alpha_{1}+2 \alpha_{2}=\beta}\left|\int_{0}^{t} \int \xi_{\delta}\left(\partial^{\alpha} u\right) \partial^{\alpha}\left(u u_{x}\right)\right| \leq C+C \sum_{\alpha_{1}+2 \alpha_{2}=\beta}\left(\int_{0}^{t} \int \xi_{\delta}\left(\partial^{\alpha} u\right)^{2}\right),
$$

where $C$ depends only on terms bounded in the previous step of the induction. Consequently, we have that

$$
\sup _{0 \leq t \leq T} \sum_{\alpha_{1}+2 \alpha_{2}=\beta} \int \xi_{\delta}\left(\partial^{\alpha} u\right)^{2}+\int_{0}^{T} \int\left(\xi_{\delta}\right)_{x}\left(\partial^{\alpha} u_{x}\right)^{2}+\int_{0}^{T} \int\left(\xi_{\delta}\right)_{x}\left(\partial^{\alpha}\left(\partial_{x}^{-1} u_{y}\right)\right)^{2} \leq C,
$$


where $C$ does not depend on $\delta$, but only on $T$ and the norm of $\varphi \in X^{0}\left(\mathbb{R}^{2}\right) \cap$ $\tilde{H}^{\beta}\left(W_{0, i, 0}\right)$. Therefore, we can pass to the limit, and get the desired estimates, namely,

$$
\sup _{0 \leq t \leq T} \sum_{\alpha_{1}+2 \alpha_{2}=\beta} \int \xi\left(\partial^{\alpha} u\right)^{2}+\int_{0}^{T} \int \eta\left(\partial^{\alpha} u_{x}\right)^{2}+\int_{0}^{T} \int \eta\left(\partial^{\alpha}\left(\partial_{x}^{-1} u_{y}\right)\right)^{2} \leq C .
$$

Now we treat the case $\beta \geq 4$.

Lemma 4.2. For $\xi_{\delta}$ as defined in (4.2), $\alpha=\left(\alpha_{1}, \alpha_{2}\right) \in \mathbb{Z}^{+} \times \mathbb{Z}^{+}$, such that $\alpha_{1}+2 \alpha_{2}=\beta, 4 \leq \beta \leq K$, the following inequality holds:

$$
\sum_{\alpha_{1}+2 \alpha_{2}=\beta}\left|\int_{0}^{t} \int \xi_{\delta}\left(\partial^{\alpha} u\right) \partial^{\alpha}\left(u u_{x}\right)\right| \leq C+C \sum_{\alpha_{1}+2 \alpha_{2}=\beta}\left(\int_{0}^{t} \int \xi_{\delta}\left(\partial^{\alpha} u\right)^{2}\right)
$$

for $0 \leq t \leq T$, where $C$ depends only on

$$
\begin{aligned}
& \sup _{0 \leq t \leq T} \int \xi_{\delta}\left(\partial^{\gamma} u\right)^{2}, \\
& \int_{0}^{T} \int\left(\xi_{\delta}\right)_{x}\left(\partial^{\gamma} u_{x}\right)^{2}, \\
& \int_{0}^{T} \int\left(\xi_{\delta}\right)_{x}\left(\partial^{\gamma}\left(\partial_{x}^{-1} u_{y}\right)\right)^{2},
\end{aligned}
$$

for $\gamma=\left(\gamma_{1}, \gamma_{2}\right) \in \mathbb{Z}^{+} \times \mathbb{Z}^{+}, \gamma_{1}+2 \gamma_{2} \leq \beta-1$.

This proof uses the same ideas as in the proof of Lemma 2.5. The primary difference is in our weight function $\xi_{\delta}$. First, our weight function here, $\xi_{\delta}$ is approximately constant for $x<-1$, where as in Lemma 2.5 , the weight function decayed exponentially for $x<-1$. Consequently, in our inductive proof of Lemma 4.2 below, we are not able to use the estimates we obtained on

$\int_{0}^{T} \int_{B}\left(\xi_{\delta}\right)_{x}\left\{\left(\partial^{\gamma} u_{x}\right)^{2}+\left(\partial^{\gamma}\left(\partial_{x}^{-1} u_{y}\right)\right)^{2}\right\} \leq C \int_{0}^{T} \int_{B} e^{\sigma x}\left\{\left(\partial^{\gamma} u_{x}\right)^{2}+\left(\partial^{\gamma}\left(\partial_{x}^{-1} u_{y}\right)\right)^{2}\right\}$

from the previous step of the induction. In addition, for $x>1$, our weight functions do not decrease by one power of $x$ on each level of the induction, as they did in Lemma 2.5 .

Proof. In order to get bounds on the left-hand side of (4.8), we use Lemma 2.6 to write every term in the integrand in the form:

$$
\xi_{\delta}\left(\partial^{\alpha} u\right)\left(\partial^{r} u\right)\left(\partial^{s} u_{x}\right)
$$


for $r_{i}+s_{i}=\alpha_{i}, i=1,2$. As in the proof of Lemma 2.5, we use the notation, $v_{r}=r_{1}+2 r_{2}, v_{s}=s_{1}+2 s_{2}$. With this notation, we have $\beta=v_{r}+v_{s}$.

Before showing the bounds on each of these terms in the integrand, we point out the following bounds. As shown in Section2,

$$
\left|\partial^{\gamma} u\right|_{L^{\infty}} \leq C\left(\int\left(\partial^{\gamma} u\right)^{2}+\left(\partial^{\gamma} u_{x x}\right)^{2}+\left(\partial^{\gamma} u_{y}\right)^{2}\right)^{1 / 2} \text {. }
$$

In addition, we can express

$$
\xi_{\delta}\left(\partial^{\gamma} u\right)=\sum_{j=0}^{\gamma_{1}}(-1)^{j+1}\left(\begin{array}{l}
\gamma \\
j
\end{array}\right) \partial_{x}^{j}\left(\left(\partial_{x}^{\gamma_{1}-j} \xi_{\delta}\right)\left(\partial_{y}^{\gamma_{2}} u\right)\right) .
$$

Combining these two facts, we know that

$$
\begin{aligned}
& \sup _{0 \leq t \leq T}\left|\xi_{\delta}\left(\partial^{\gamma} u\right)^{2}\right|_{L^{\infty}} \leq C \text { for } v \leq \beta-3, \\
& \int_{0}^{T}\left|\left(\xi_{\delta}\right)_{x}\left(\partial^{\gamma} u\right)^{2}\right|_{L^{\infty}} \leq C \text { for } v \leq \beta-2,
\end{aligned}
$$

where $\gamma_{1}+2 \gamma_{2}=v$ and $C$ depends only on (4.9)-(4.11). We will use estimates (4.12) and (4.13) below in bounding each term in the integrand. As we did in Lemma 2.5, we will look at the various cases separately.

$\triangleright$ The case $v_{s} \leq \beta-4$. For the case $v_{s} \leq \beta-4$, we have

$$
\begin{aligned}
& \left|\int_{0}^{t} \int \xi_{\delta}\left(\partial^{\alpha} u\right)\left(\partial^{r} u\right)\left(\partial^{s} u_{x}\right)\right| \\
& \quad \leq \sup _{0 \leq t \leq T}\left|\xi_{\delta}^{1 / 2}\left(\partial^{s} u_{x}\right)\right|_{L^{\infty}}\left(\int_{0}^{t} \int\left(\partial^{r} u\right)^{2}\right)^{1 / 2}\left(\int_{0}^{t} \int \xi_{\delta}\left(\partial^{\alpha} u\right)^{2}\right)^{1 / 2} .
\end{aligned}
$$

In this case, the first term is bounded by (4.12). If $v_{r} \leq \beta-1$, then the second term is bounded by (4.9). If $\nu_{r}=\beta$, then the second term is bounded by $\sum_{\alpha_{1}+2 \alpha_{2}=\beta}\left(\int_{0}^{t} \int \xi_{\delta}\left(\partial^{\alpha} u\right)^{2}\right)^{1 / 2}$. In either case, we have

$$
\left|\int_{0}^{t} \int \xi_{\delta}\left(\partial^{\alpha} u\right)\left(\partial^{r} u\right)\left(\partial^{s} u_{x}\right)\right| \leq C+C \sum_{\alpha_{1}+2 \alpha_{2}=\beta}\left(\int_{0}^{t} \int \xi_{\delta}\left(\partial^{\alpha} u\right)^{2}\right)
$$

for $v_{s} \leq \beta-4$, as desired.

$\triangleright$ The case $\beta-4<v_{s} \leq \beta-2$. For this case, we will consider $x>1$ and $x<-1$ separately, because of the nature of the weight function. For $x>1$, $\xi_{\delta} \leq C x^{L}$, while for $x<-1, \xi_{\delta} \approx C\left(1+e^{\sigma x}\right)$. Again, let $A=\{x>1\} \times \mathbb{R}$ and $B=\{x<-1\} \times \mathbb{R}$. 
$\triangleright$ The subcase $x>1$. First, for $x>1$, if $\beta \geq 5$, we can use the following estimate,

$$
\begin{aligned}
&\left|\int_{0}^{t} \int_{A} \xi_{\delta}\left(\partial^{\alpha} u\right)\left(\partial^{r} u\right)\left(\partial^{s} u_{x}\right)\right| \leq\left(\int_{0}^{t}\left|\left(\xi_{\delta}\right)_{x}\left(\partial^{r} u\right)^{2}\right|_{L^{\infty}(A)}\right)^{1 / 2} \\
& \cdot\left(\sup _{0 \leq t \leq T} \int_{A} \xi_{\delta}\left(\partial^{s} u_{x}\right)^{2}\right)^{1 / 2}\left(\int_{0}^{t} \int_{A} \xi_{\delta}\left(\partial^{\alpha} u\right)^{2}\right)^{1 / 2} .
\end{aligned}
$$

The first term is bounded by (4.13) because $v_{r} \leq \beta-2$. The second term is bounded by (4.9) because $v_{s} \leq \beta-2$. We can use that same estimate for the case $\beta=4, v_{s}=\beta-2$.

If $\beta=4$ and $v_{s}=\beta-3$, we have $v_{s}=1, v_{r}=3$, in which case,

$$
\begin{aligned}
& \left|\int_{0}^{t} \int_{A} \xi_{\delta}\left(\partial^{\alpha} u\right)\left(\partial^{r} u\right)\left(\partial^{s} u_{x}\right)\right| \\
& \quad=\left|\int_{0}^{t} \int_{A} \xi_{\delta} u_{x x}\left(\partial^{r} u\right)\left(\partial^{\alpha} u\right)\right| \\
& \quad \leq\left(\int_{0}^{t}\left|\left(\xi_{\delta}\right)_{x} u_{x x}^{2}\right|_{L^{\infty}(A)}\right)^{1 / 2}\left(\sup _{0 \leq t \leq T} \int_{A} \xi_{\delta}\left(\partial^{r} u\right)^{2}\right)^{1 / 2}\left(\int_{0}^{t} \int_{A} \xi_{\delta}\left(\partial^{\alpha} u\right)^{2}\right)^{1 / 2} .
\end{aligned}
$$

The first term is bounded by (4.13) because $v_{s}=2 \leq \beta-2$. The second term is bounded by (4.9) because $v_{r} \leq \beta-1$.

Remark. Notice that we cannot use the same estimate when $x<-1$, because for $x<-1, \xi_{\delta} \approx C$, which means that when we divide our weight function amongst the three other functions, each function still has a constant function as a weight. In particular, this means we cannot use estimate (4.13) as we did above. Specifically, $\xi_{\delta}^{1 / n} \npreceq C\left(\xi_{\delta}\right)_{x}$ for any $n>0, x<-1$.

$\triangleright$ The subcase $x<-1$. For the case $x<-1, \xi_{\delta} \approx 1+e^{\sigma x} \leq C$. For $v_{s}=\beta-3, \beta \geq 6$ or $v_{s}=\beta-2, \beta \geq 5$, we can bound as follows

$$
\begin{aligned}
& \left|\int_{0}^{t} \int_{B} \xi_{\delta}\left(\partial^{\alpha} u\right)\left(\partial^{r} u\right)\left(\partial^{s} u_{x}\right)\right| \\
\leq & C\left(\sup _{0 \leq t \leq T}\left|\left(\xi_{\delta}\right)^{1 / 2}\left(\partial^{r} u\right)\right|_{L^{\infty}(B)}\right)\left(\int_{0}^{t} \int_{B} \xi_{\delta}\left(\partial^{s} u_{x}\right)^{2}\right)^{1 / 2}\left(\int_{0}^{t} \int_{B} \xi_{\delta}\left(\partial^{\alpha} u\right)^{2}\right)^{1 / 2} .
\end{aligned}
$$

The first term is bounded by (4.12) because $v_{r} \leq \beta-3$. The second term is bounded by (4.9) because $v_{s} \leq \beta-2$. 
Next we consider the case $\beta=5, v_{s}=\beta-3$.

$$
\begin{gathered}
\left|\int_{0}^{t} \int_{B} \xi_{\delta}\left(\partial^{\alpha} u\right)\left(\partial^{r} u\right)\left(\partial^{s} u_{x}\right)\right| \\
\leq C\left(\int_{0}^{t} \int_{B}\left(\partial^{r} u\right)^{4}\right)^{1 / 4}\left(\int_{0}^{t} \int_{B}\left(\partial^{s} u_{x}\right)^{4}\right)^{1 / 4}\left(\int_{0}^{t} \int_{B}\left(\partial^{\alpha} u\right)^{2}\right)^{1 / 2} \\
\leq C\left(\int_{0}^{t} \int_{B} \xi_{\delta}\left\{\left(\partial^{r} u\right)^{2}+\left(\partial^{r} u_{x}\right)^{2}+\left(\partial^{r}\left(\partial_{x}^{-1} u_{y}\right)\right)^{2}\right\}\right)^{1 / 2} \\
\cdot\left(\int_{0}^{t} \int_{B} \xi_{\delta}\left\{\left(\partial^{s} u_{x}\right)^{2}+\left(\partial^{s} u_{x x}\right)^{2}+\left(\partial^{s} u_{y}\right)^{2}\right\}\right)^{1 / 2} \\
\cdot\left(\int_{0}^{t} \int_{B} \xi_{\delta}\left(\partial^{\alpha} u\right)^{2}\right)^{1 / 2}
\end{gathered}
$$

using (3.7) on the first two terms on the right-hand side. The first and second terms are each bounded by (4.9) because $\nu_{r}=\beta-2$ and $\nu_{s}=\beta-3$.

Lastly, for $x<-1$, we consider $\beta=4$. First, if $v_{s}=\beta-3$, then we have,

$$
\begin{aligned}
& \left|\int_{0}^{t} \int_{B} \xi_{\delta}\left(\partial^{\alpha} u\right)\left(\partial^{r} u\right)\left(\partial^{s} u_{x}\right)\right| \\
& \leq C\left(\int_{0}^{t}\left|u_{x x}\right|_{L^{\infty}(B)}^{2}\right)\left(\sup _{0 \leq t \leq T} \int_{B}\left(\partial^{r} u\right)^{2}\right)^{1 / 2}\left(\int_{0}^{t} \int_{B}\left(\partial^{\alpha} u\right)^{2}\right)^{1 / 2} \\
& \leq C\left(\int_{0}^{t} \int_{B} \xi_{\delta}\left\{u_{x x}^{2}+u_{x x x x}^{2}+u_{x x y}^{2}\right\}\right)^{1 / 2}\left(\sup _{0 \leq t \leq T} \int_{B} \xi_{\delta}\left(\partial^{r} u\right)^{2}\right)^{1 / 2} \\
& \quad \cdot\left(\int_{0}^{t} \int_{B} \xi_{\delta}\left(\partial^{\alpha} u\right)^{2}\right)^{1 / 2} \\
& \leq C+C \sum_{\alpha_{1}+2 \alpha_{2}=4}\left(\int_{0}^{t} \int_{B} \xi_{\delta}\left(\partial^{\alpha} u\right)^{2}\right) .
\end{aligned}
$$

Second, if $v_{s}=\beta-2$, we have $v_{r}=v_{s}=2$, in which case, we have four possibilites. We will take care of each of these below. First, consider $r=$ 
$(2,0), s=(2,0)$, in which case

$$
\begin{aligned}
& \left|\int_{0}^{t} \int_{B} \xi_{\delta}\left(\partial^{\alpha} u\right)\left(\partial^{r} u\right)\left(\partial^{s} u_{x}\right)\right| \\
& \quad \leq C\left|\int_{0}^{t} \int_{B} u_{x x} u_{x x x} u_{x x x x}\right| \\
& \quad \leq\left(\sup _{0 \leq t \leq T} \int_{B} u_{x x x}^{2}\right)^{1 / 2}\left(\int_{0}^{t}\left|u_{x x}\right|_{L^{\infty}(B)}^{2}\right)^{1 / 2}\left(\int_{0}^{t} \int_{B} u_{x x x x}^{2}\right)^{1 / 2} \\
& \quad \leq C\left(\int_{0}^{t} \int_{B} u_{x x}^{2}+u_{x x x x}^{2}+u_{x x y}^{2}\right)^{1 / 2}\left(\int_{0}^{t} \int_{B} u_{x x x x}^{2}\right)^{1 / 2} \\
& \quad \leq C\left(\int_{0}^{t} \int_{B} \xi_{\delta}\left\{u_{x x x}^{2}+u_{x x x x}^{2}+u_{x x y}^{2}\right\}\right) .
\end{aligned}
$$

Similarly, if $r=(2,0), s=(0,1)$,

$$
\begin{aligned}
& \left|\int_{0}^{t} \int_{B} \xi_{\delta}\left(\partial^{\alpha} u\right)\left(\partial^{r} u\right)\left(\partial^{s} u_{x}\right)\right| \\
& \quad \leq C\left|\int_{0}^{t} \int_{B} u_{x x} u_{x y} u_{x x y}\right| \\
& \quad \leq\left(\sup _{0 \leq t \leq T} \int_{B} u_{x y}^{2}\right)^{1 / 2}\left(\int_{0}^{t}\left|u_{x x}\right|_{L^{\infty}(B)}^{2}\right)^{1 / 2}\left(\int_{0}^{t} \int_{B} u_{x x y}^{2}\right)^{1 / 2} \\
& \quad \leq C\left(\int_{0}^{t} \int_{B} u_{x x}^{2}+u_{x x x x}^{2}+u_{x x y}^{2}\right)^{1 / 2}\left(\int_{0}^{t} \int_{B} u_{x x y}^{2}\right)^{1 / 2} \\
& \quad \leq C\left(\int_{0}^{t} \int_{B} \xi_{\delta}\left\{u_{x x}^{2}+u_{x x x x}^{2}+u_{x x y}^{2}\right\}\right) .
\end{aligned}
$$

For the case, $r=(0,1), s=(2,0)$, we have:

$$
\begin{aligned}
& \left|\int_{0}^{t} \int_{B} \xi_{\delta}\left(\partial^{\alpha} u\right)\left(\partial^{r} u\right)\left(\partial^{s} u_{x}\right)\right| \\
& \quad \leq C\left|\int_{0}^{t} \int_{B} u_{x x x} u_{y} u_{x x y}\right| \\
& \quad \leq C\left(\sup _{0 \leq t \leq T} \int_{B} u_{x x x}^{2}\right)^{1 / 2}\left(\int_{0}^{t}\left|u_{y}\right|_{L^{\infty}(B)}^{2}\right)\left(\int_{0}^{t} \int_{B} u_{x x y}^{2}\right)^{1 / 2} \\
& \quad \leq C\left(\int_{0}^{t} \int_{B} u_{y}^{2}+u_{x x y}^{2}+u_{y y}^{2}\right)^{1 / 2}\left(\int_{0}^{t} \int_{B} u_{x x y}^{2}\right)^{1 / 2} \\
& \quad \leq C\left(\int_{0}^{t} \int_{B} \xi_{\delta}\left\{u_{y}^{2}+u_{x x y}^{2}+u_{y y}^{2}\right\}\right) .
\end{aligned}
$$


Similarly, for $r=(0,1), s=(0,1)$,

$$
\begin{aligned}
& \left|\int_{0}^{t} \int_{B} \xi_{\delta}\left(\partial^{\alpha} u\right)\left(\partial^{r} u\right)\left(\partial^{s} u_{x}\right)\right| \\
& \quad \leq C\left|\int_{0}^{t} \int_{B} u_{x y} u_{y} u_{y y}\right| \\
& \quad \leq\left(\sup _{0 \leq t \leq T} \int_{B} u_{x y}^{2}\right)^{1 / 2}\left(\int_{0}^{t}\left|u_{y}\right|_{L^{\infty}(B)}^{2}\right)^{1 / 2}\left(\int_{0}^{t} \int_{B} u_{y y}^{2}\right)^{1 / 2} \\
& \quad \leq C\left(\int_{0}^{t} \int_{B} u_{y}^{2}+u_{x x y}^{2}+u_{y y}^{2}\right)^{1 / 2}\left(\int_{0}^{t} \int_{B} u_{y y}^{2}\right)^{1 / 2} \\
& \quad \leq C\left(\int_{0}^{t} \int_{B} \xi_{\delta}\left\{u_{y}^{2}+u_{x x y}^{2}+u_{y y}^{2}\right\}\right) .
\end{aligned}
$$

Combining these inequalities, we conclude that

$$
\sum_{\alpha_{1}+2 \alpha_{2}=4}\left|\int_{0}^{t} \int \xi_{\delta}\left(\partial^{\alpha} u\right) \partial^{\alpha}\left(u u_{x}\right)\right| \leq C+C \sum_{\alpha_{1}+2 \alpha_{2}=4}\left(\int_{0}^{t} \int \xi_{\delta}\left(\partial^{\alpha} u\right)^{2}\right),
$$

as desired.

$\triangleright$ The case $\beta \geq v_{s} \geq \beta-1$. Lastly, we consider the cases $v_{s}=\beta-1$ and $v_{s}=\beta$. If $v_{s}=\beta-1$, then $v_{r}=1$ and we have

$$
\begin{aligned}
\left|\int_{0}^{t} \int \xi_{\delta}\left(\partial^{\alpha} u\right)\left(\partial^{r} u\right)\left(\partial^{s} u_{x}\right)\right| & =\left|\int_{0}^{t} \int \xi_{\delta} u_{x}\left(\partial^{\alpha} u\right)^{2}\right| \\
& \leq C\|u\|_{X^{0}}\left(\int_{0}^{t} \int \xi_{\delta}\left(\partial^{\alpha} u\right)^{2}\right) .
\end{aligned}
$$

Similarly, if $v_{s}=\beta$, then $v_{r}=0$ and

$$
\begin{aligned}
\left|\int_{0}^{t} \int \xi_{\delta}\left(\partial^{\alpha} u\right)\left(\partial^{r} u\right)\left(\partial^{s} u_{x}\right)\right| & =\left(\int_{0}^{t} \int\left[\xi_{\delta} u\right]_{x}\left(\partial^{\alpha} u\right)^{2}\right) \\
& \leq C\|u\|_{X^{0}}\left(\int_{0}^{t} \int \xi_{\delta}\left(\partial^{\alpha} u\right)^{2}\right) .
\end{aligned}
$$

We now have all the components to give a proof of our main theorem on the gain of regularity for the KP-II equation. 


\section{Proof of Theorem 2.1.}

By assumption, $u \in L^{\infty}\left([0, T] ; Z_{L}\right)$. Recall this means $u \in X^{0}\left(\mathbb{R}^{2}\right)$ and $\int \xi\left(u^{2}+u_{x}^{2}\right)<+\infty$ for $\xi \in W_{0, L, 0}$. The equation implies $u_{t} \in$ $L^{\infty}\left([0, T] ; L^{2}\left(\mathbb{R}^{2}\right)\right)$. Therefore, $u$ is a weakly continuous function of $t$ with values in $Z_{L}$, and, in particular, $u(\cdot, t)$ is in $Z_{L}$ for every $t$. Let $\left\{\varphi^{(n)}\right\}$ be a sequence of functions in $C_{0}^{\infty}\left(\mathbb{R}^{2}\right)$ such that $\partial_{x}^{-1} \varphi_{y y}^{(n)}$ are in $C_{0}^{\infty}$, which converges to $u\left(\cdot, t_{0}\right)$ strongly in $Z_{L}$, for $0 \leq t_{0}<T$. Let $u^{(n)}$ be the unique solution of (2.1) with initial data $\varphi^{(n)}(x, y)$ at time $t=t_{0}$. By Corollary 3.6, the solution $u^{(n)} \in L^{\infty}\left(\left[t_{0}, t_{0}+\delta\right] ; X^{0}\left(\mathbb{R}^{2}\right)\right)$ for a time interval $\delta$ which does not depend on $n$. By Theorem 4.1, $u^{(n)} \in L^{\infty}\left(\left[t_{0}, t_{0}+\delta\right] ; Z_{L}\right)$ and

$$
\begin{array}{r}
\int_{t_{0}}^{t_{0}+\delta} \int \eta\left(u_{x}^{(n)}\right)^{2} d x d y d t+\int_{t_{0}}^{t_{0}+\delta} \int \eta\left(\partial_{x}^{-1} u_{y}^{(n)}\right)^{2} d x d y d t \leq C \\
\int_{t_{0}}^{t_{0}+\delta} \int \eta\left(u_{x x}^{(n)}\right)^{2} d x d y d t+\int_{t_{0}}^{t_{0}+\delta} \int \eta\left(u_{y}^{(n)}\right)^{2} d x d y d t \leq C
\end{array}
$$

where $\eta \in W_{\sigma, L-1,0}$ and $C$ depends only on the norm of $\varphi^{(n)} \in Z_{L}$. Also by Theorem 4.1, we have (non-uniform) bounds on

$$
\sup _{\left[t_{0}, t_{0}+\delta\right]} \sup _{x}\left(1+x_{+}\right)^{k}\left|\partial^{\alpha} u^{(n)}(x, y, t)\right|<+\infty
$$

for each $n, k$, and $\alpha$. Therefore, the a priori estimates in Lemma 2.2 are justified for each $u^{(n)}$ in the interval $\left[t_{0}, t_{0}+\delta\right]$.

We start our induction with $\beta=2$, in which case $\alpha=(2,0)$ or $\alpha=(0,1)$. Take $\eta \in W_{\sigma, L-2,2}$ and let $\xi=\int_{-\infty}^{x} \eta(z, t) d z$. As shown in Lemma 2.2, we have the following bounds on the higher derivatives of $u^{(n)}$,

$$
\sup _{\left[t_{0}, t_{0}+\delta\right]} \int \xi\left(u_{x x}^{(n)}\right)^{2}+\int_{t_{0}}^{t_{0}+\delta} \int \eta\left(u_{x x x}^{(n)}\right)^{2}+\int_{t_{0}}^{t_{0}+\delta} \int \eta\left(u_{x y}^{(n)}\right)^{2} \leq C,
$$

(4.18) $\sup _{\left[t_{0}, t_{0}+\delta\right]} \int \xi\left(u_{y}^{(n)}\right)^{2}+\int_{t_{0}}^{t_{0}+\delta} \int \eta\left(u_{x y}^{(n)}\right)^{2}+\int_{t_{0}}^{t_{0}+\delta} \int \eta\left(\partial_{x}^{-1} u_{y y}^{(n)}\right)^{2} \leq C$,

where $C$ depends only on the norm of $u^{(n)} \in L^{\infty}\left([0, T] ; Z_{L}\right)$ and the terms in (4.14) and (4.15). We conclude, therefore, that the constant $C$ in (4.17) depends only on $\left\|\varphi^{(n)}\right\| z_{z_{L}}$.

We continue this procedure inductively. For the $\beta^{\text {th }}$ step, let

$$
\eta \in W_{\sigma, L-(\beta-1)^{+-}-1,(\beta-1)^{+},}
$$


and define $\xi=\int_{-\infty}^{x} \eta(z, t) d z$. The non-uniform bounds on $u^{(n)}$ in (4.16) allow us to use Lemma 2.2and our inductive hypothesis to conclude that

$$
\sup _{\left[t_{0}, t_{0}+\delta\right]} \int \xi\left(\partial^{\alpha} u^{(n)}\right)^{2}+\int_{t_{0}}^{t_{0}+\delta} \int \eta\left(\partial^{\alpha} u_{x}^{(n)}\right)^{2}+\int_{t_{0}}^{t_{0}+\delta} \int \eta\left(\partial^{\alpha}\left(\partial_{x}^{-1} u_{y}^{(n)}\right)\right)^{2} \leq C,
$$

where again $C$ does not depend on $n$, but only on the norm of $\varphi^{(n)} \in Z_{L}$. By Corollary 3.7, $u^{(n)} \rightarrow u$ weak $^{*}$ in $L^{\infty}\left(\left[t_{0}, t_{0}+\delta\right] ; X^{0}\left(\mathbb{R}^{2}\right)\right)$, therefore, we can pass to the limit and conclude that:

$$
\sup _{\left[t_{0}, t_{0}+\delta\right]} \int \xi\left(\partial^{\alpha} u\right)^{2}+\int_{t_{0}}^{t_{0}+\delta} \int \eta\left(\partial^{\alpha} u_{x}\right)^{2}+\int_{t_{0}}^{t_{0}+\delta} \int \eta\left(\partial^{\alpha}\left(\partial_{x}^{-1} u_{y}\right)\right)^{2} \leq C .
$$

This proof is continued inductively up to $\beta=L+1$. Since $\delta$ does not depend on $n$, this result if valid over the whole interval $[0, T]$.

\section{REFERENCES}

[AC] M. J. Ablowitz \& P. A. Clarkson, Solitons, Nonlinear Evolution Equations and Inverse Scattering, Cambridge University Press, Cambridge, 1991.

[BIN] O. V. BESOV, V. P. IL'IN \&. S. M. NIKOLSKII, Integral Representations of Functions and Imbedding Theorems, Vol. I, J. Wiley, 1978.

[Bo] J. Bourgain, On the Cauchy problem for the Kadomtsev-Petviashvili equation, Geom. Funct. Anal. 3 (1993), 315-341.

[Co] A. CoHEn, Solutions of the Korteweg-de Vries equation from irregular data, Duke Math. J. 45 (1978), 149-181.

[CG] W. CRAig \& J. GoOdman, Linear dispersive equations of airy type, J. Diff. Eqns. 7 (1990), 38-61.

[CKS] W. CRAig, T. Kappeler \& W. STRAuss, Gain of regularity for equations of $K d V$ type, Ann. Inst. H. Poincare 9 (1992), 147-186.

[DLW] D. David, D. LeVI \& P. Winternitz, Solitons in shallow seas of variable depth and in marine straits, Stud. Appl. Math. 80 (1989), 1-23.

[Fa] A. V. FAMINSKII, The Cauchy problem for the generalized Kadomtsev-Petviashvili equation, Sibirsk. Mat. Zh. 33 (1992), 160-172.

[Ka] T. KATO, On the Cauchy problem for the (generalized) Korteweg-de Vries equation, Adv. in Math. Suppl. Studies: Studies in Appl. Math. 8 (1983), 93-128.

[KP] B. B. KadomtseV \& V. I. PETViashvili, On the stability of solitary waves in weakly dispersive media, Sov. Phys. Dokl. 15 (1970), 539-541.

[PY] V. I. Petviashvili \& V. V. YAN'KOv, Solitons and turbulence, Rev. Plasma Phys., XIV (1989), 1-62.

[Sa1] J. C. SAUT, Recent results on the generalized Kadomtsev-Petviashvili equations. KdV '95, Acta Appl. Math. 39 (1995), 477-487.

[Sa2] J. C. SAUT, Remarks on the generalized Kadomtsev-Petviashvili equations, Ind. Univ. Math. J. 42 (1993), 1011-1026. 
[Sn] P. M. SANTINI, On the evolution of two-dimensional packets of water waves over an uneven bottom, Lett. Nuovo Cim. 30 (1981), 236-240.

[Uk] S. UKAI, Local solutions of the Kadomtsev-Petviashvili equation, J. Fac. Sci. Univ. Tokyo Sect. 1A Math. 36 (1989), 193-209.

Acknowledgment. This research was supported in part by National Science Foundation Grant DMS 97-03695.

JULIE L. LEVANDOSKI

Department of Mathematics

Brown University

Providence, RI 02912

CURRENT AdDress

Department of Mathematics

Stanford University

Stanford, CA 94305

EMAIL: julie@math.stanford.edu

SubJeCt ClassificATION: 35Q53

KEYWORDS: KP equation, dispersive equations

Submitted: October 27th, 1998; revised: June 10th, 1999. 